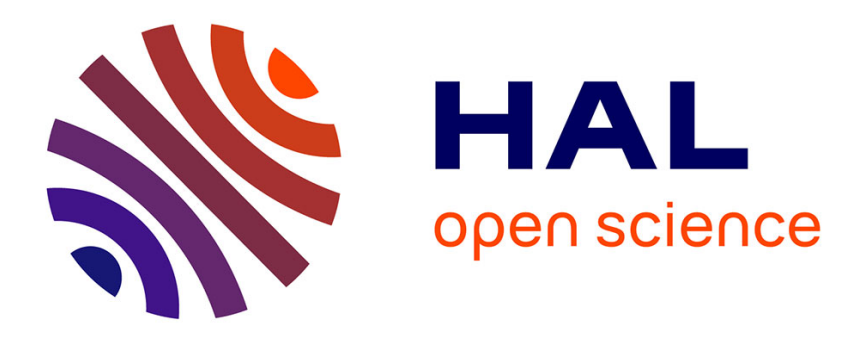

\title{
Buckling of Naturally Curved Elastic Strips: The Ribbon Model Makes a Difference
}

Basile Audoly, Keith A. Seffen

\section{To cite this version:}

Basile Audoly, Keith A. Seffen. Buckling of Naturally Curved Elastic Strips: The Ribbon Model Makes a Difference. Journal of Elasticity, 2015, pp.1-1. 10.1007/s10659-015-9520-y . hal-01128595

\section{HAL Id: hal-01128595 \\ https://hal.science/hal-01128595}

Submitted on 10 Mar 2015

HAL is a multi-disciplinary open access archive for the deposit and dissemination of scientific research documents, whether they are published or not. The documents may come from teaching and research institutions in France or abroad, or from public or private research centers.
L'archive ouverte pluridisciplinaire HAL, est destinée au dépôt et à la diffusion de documents scientifiques de niveau recherche, publiés ou non, émanant des établissements d'enseignement et de recherche français ou étrangers, des laboratoires publics ou privés. 


\title{
Buckling of naturally curved elastic strips: the ribbon model makes a difference
}

\author{
Basile Audoly*1 and Keith A. Seffen ${ }^{\dagger 2}$ \\ ${ }^{1}$ Sorbonne Universités, UPMC Univ Paris 06, CNRS, UMR 7190 \\ Institut Jean Le Rond d'Alembert, F-75005 Paris, France \\ ${ }^{2}$ Department of Engineering, University of Cambridge, \\ Trumpington Street, Cambridge CB2 1PZ, UK
}

March 10, 2015

\begin{abstract}
We analyze the stability of naturally curved, inextensible elastic ribbons. In experiments, we first show that a loop formed using a metallic strip can become unstable if its radius is smaller than its natural radius of curvature (undercurved case): the loop then folds onto itself into a smaller, multiply-covered loop. Conversely, a multi-covered, overcurved metallic strip can unfold dynamically into a circular configuration having a lower covering index. We analyze these instabilities using a one-dimensional mechanical model for an elastic ribbon introduced recently (Dias Audoly, 2014), which extends Sadowsky's developable elastic ribbon model in the presence of natural curvature. Combining linear stability analyses and numerical computations of the post-buckled configurations, we classify the equilibria of the ribbon as a function of the ratio of its natural curvature to its actual curvature. Our ribbon model is formulated in close analogy with classical rod models; this allows us to adapt classical stability methods for rods to the case of a ribbon. The stability of a ribbon is found to differ significantly from that of an anisotropic rod: we attribute this difference to the fact that the tangent twisting modulus of a ribbon can be negative, in contrast to what is possible in the well-studied case of linearly elastic rods. The specific stability properties predicted by the curved ribbon model are confirmed by a finite element analysis of cylindrical shells having a small height-to-radius ratio.
\end{abstract}

Keywords: Elastic plates 74K20, Elastic rods 74K10, Energy minimization $74 \mathrm{G} 65$

*audoly@lmm.jussieu.fr

†kas14@cam.ac.uk 


\section{Introduction}

As their cross-section is much smaller than their length, slender rods can undergo significant rotations while the material strains remain in the elastic range. The stability of thin elastic rods has long been recognized as an important issue, and has developed into a vast, and now classical field [1. To give just two examples, excessive deformation of slender rods can describe the failure of engineering components such as drill strings, masts and pipelines 24 as well as the conformations of biological molecules, in particular, DNA [12]. Thin rods are usually considered capable of bending about two orthogonal axes and twisting along their centerlines, where natural curvature and torsion can be specified initially to capture different starting geometries. Three imperatives underpin the analysis of deformation: equilibrium, compatibility and material constitutive behavior in the form of generalized Hooke's laws; and for finite rotations, these become coupled within a nonlinear framework, for example, the well-known Kirchhoff rod equations of force and moment equilibrium on a given cross-section.

The cross-sectional shape of a rod determines its bending and torsional stiffness about its principal axes. For linearly elastic rods, these laws are linear themselves. When the cross-section tends towards being flat, i.e. when the rod is more like a strip or ribbon, a beam-like viewpoint merely adjusts the stiffnesses according to their geometrical definitions. For a very thin ribbon, the stiffness ratio may be very large, which affects the physical deformation in some dominant way and, more importantly, the integrity of the assumptions on which the beam theory is founded. It is therefore imperative to reformulate the assumptions of cross-sectional influence. Typically, researchers assume that the ribbon behaves like a narrow and thin elastic plate or shell [20, 21, 22]. Care needs to be exercised in the context of highly deformed strips, where the levels of local deformation are large enough to warrant a developable response in which the Gaussian curvature all but disappears; this imposes further constraint upon the kinematical relationships between curvature and torsion.

To date, the two approaches to thin elastic strips have been developed separately, each one having its own advantages and drawbacks. On one hand, modelling strips as anisotropic rods makes it possible to reuse the large body of work available on the analysis and computation of equilibria, stability and dynamics of thin rods; however, accuracy can be compromised because rod models ignore the special role played by developable configurations of the midsurface of a thin strip. Such configurations have a much lower elastic energy than nondevelopable ones when the thickness is much smaller than the width. On the other hand, ribbon models based on plate theory, which appeared in the seminal works of Sadowsky [20, 19, 9, 10] and Wunderlich [26, 25, are mechanically accurate but were developed as a largely independent branch of elasticity. This branch is much less advanced - for instance, the equations of equilibrium of a ribbon were not derived until relatively recently [22], and no stability analysis based on the one-dimensional ribbon model has been carried out to date, to the best of our knowledge. 
In this paper, we try to bring together the best of both worlds, and show that the ribbon model, when appropriately formulated, can be analyzed in close analogy to a rod model, without losing physical accuracy. To this end, we use the ribbon model introduced by Dias and Audoly in another paper of this special issue [3, which extends both the energy proposed by Sadowsky [20] and the equations of equilibrium derived by Starostin and van der Heijden [22] for a flat ribbon, to a naturally curved ribbon. This curved ribbon model is formulated mathematically as a constrained elastic rod: like rods, but unlike other ribbon models, it makes use of a frame of directors; in this model, the inextensibility ${ }^{1}$ of the midsurface of the ribbon is taken into account by specifying kinematical constraints, which gives rise to an effectively nonlinear constitutive law 3]. Being expressed in the same language as an elastic rod model, the ribbon model of [3] enables us in the present paper $(i)$ to set up a stability analysis for a onedimensional ribbon model for the first time, by a straightforward adaptation of the classical stability methods used for rods, and (ii) to carry out a detailed comparison of the predictions of the rod and ribbon models.

To illustrate this ideas, we consider a specific geometry, namely the buckling instabilities of simple or multi-covered loops made of a naturally elastic ribbon, when there is a mismatch between their natural radius of curvature and their actual radius of curvature. In previous work, this problem has been addressed using rod models including the effect of cross-section anisotropy and natural curvature [7, 6, 15]; as our paper shows, the ribbon model yields markedly different results. This choice of geometry is inspired by physical models with ribbon-like cross-sections encountered during our research; industrial bandsaw blades packaged as continuous loops of three turns and foldable sun-shades for protecting vehicle passengers from sideways sunlight, both of which unfold into single loop, planar structures. Both have initial, or "intrinsic" curvature, which is revealed when the loop is severed; the ribbon has a different, unstressed natural curvature. By reconnecting the original loop, the ribbon is loaded internally and acquires pre-stress, and the levels of pre-stress and, hence, intrinsic curvature dictate that each loop can be uniquely folded into three planar hoops. The stability of this configuration relative to the open-loop state is studied here for each of the different cross-sectional models, with the objective of better understanding the influences of each set of assumptions. We find that stable loops with more than three turns are also possible, and we build a physical demonstrator.

The layout of this paper is as follows. Section 2 details our construction and testing of a multistable hoop from a bandsaw blade. This blade originally has stable single and triple ring states, and we adjust the natural curvature plastically to explore the effect upon stability; we determine the values at which the stability is lost or gained, leading us to a novel configuration of five stable rings. These results are later compared to theoretical values in Section 6, and we also describe a simple procedure for reliably folding the hoop between states.

\footnotetext{
${ }^{1}$ An inextensible surface is a surface that preserves its metric, hence the length of all curves drawn onto it, upon deformation. We do not consider the case of surfaces that are inextensible along a given set of material directions only, such as surfaces made up of woven networks of inextensible fibers.
} 
Section 3 introduces our three modelling scenarios: the isotropic rod, the flat rod and the ribbon. All of them assume inextensibility along the tangent line normal to each cross-section, which we impose via a constrained Cosserat framework. The constitutive, or generalized Hooke's, laws relating the internal moment and curvature-twist components on a given cross-section to each other are linear for the rod models and non-linear for the ribbon model. In Section 4 all of these laws are imported into the equations of motion linearized about initially circular configurations, so that we may begin to understand later the differences in properties of the multistable hoop. A key difference between the ribbon and rod models is identified at this stage: the tangent twisting modulus of both rod models is constant and positive, although that of the ribbon model varies and may be negative. In section 5 , the consequence of the positiveness or nonpositiveness of this modulus is explored in a simple scenario of the unbending of an initially curved rod: we find that the ribbon model becomes unstable close to the point where its tangent twisting modulus passes through zero, but the rod models remain stable until a considerably larger deformation is applied. We carry out a finite element analysis of a shell model to confirm the predictions of the ribbon model. In section 6, we return to the original problem of the stability of the multiply-covered strips, which we approach analytically and computationally using the ribbon model. We present a combination of dynamic stability analyses and numerical simulations of post-buckled equilibrium shapes. A global picture of the equilibria and their stability emerges, which is both consistent with our experiments and different from previous predictions based on rod models.

\section{Experiments}

Figure 1 shows a loop constructed from a $3 \mathrm{~m}$ length of bandsaw blade whose teeth have been ground down for ease of handling, resulting in a rectangular cross-section of width $5.65 \mathrm{~mm}$ and thickness $0.65 \mathrm{~mm}$. The blade was passed in a controlled fashion through a set of rollers to impart uniform intrinsic curvature, $\kappa_{\mathrm{n}}$, about the minor axis of bending of cross-section, and the final open section has around 3.33 turns as indicated, of radius $r_{0} \approx 143 \mathrm{~mm}$. The ends of the blade are then connected together by a short bracket to form a continuous structure of three loops, which can be unfolded into a single loop or folded into five- and seven loops, as we soon describe. Apart from the single loop, each folded shape is almost but not quite planar owing to interference during the overlapping of turns; all have circular loops of the same radius and, crucially, the size of the initial curvature imparted by rolling allows each configuration to be locally stable, i.e. to be relatively insensitive to sufficiently small out-of-plane perturbations.

Folding and obtaining each form is straightforward after some practice. Starting from a single loop, the ribbon may be twisted manually along any diameter in the out-of-plane direction, as displayed in Fig. 2, until it drives itself into three equal loops. To obtain five loops, the radius of one of the three 


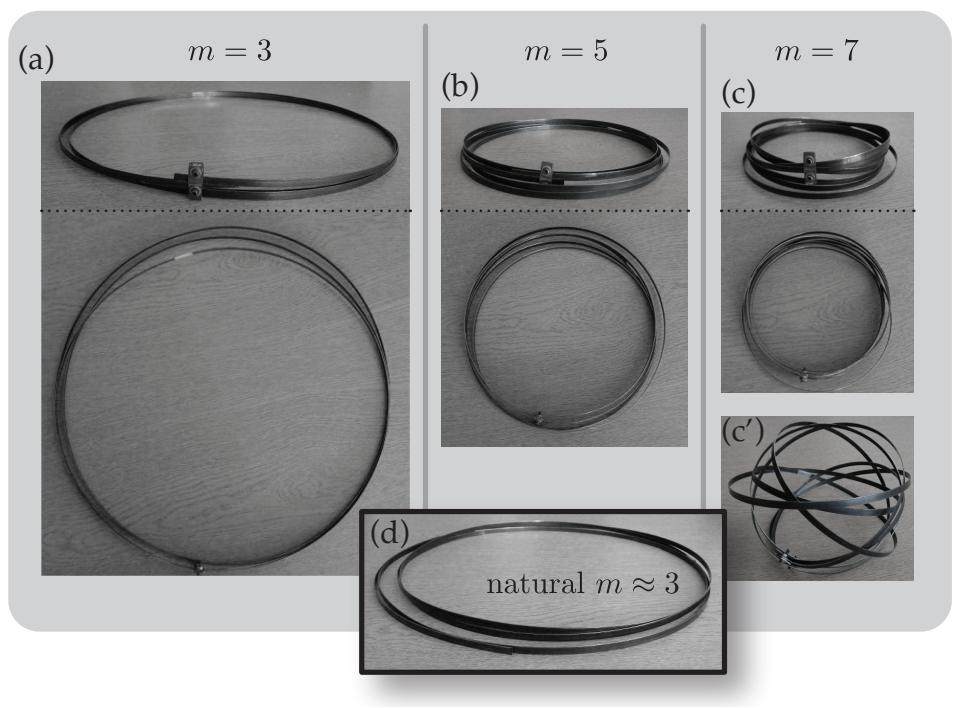

Figure 1: Stable configurations of a bandsaw blade performing as a multistable hoop. The usual teeth have been removed from a commercially available product of initial length of around $3 \mathrm{~m}$. The blade comes with two stable shapes; the open, single loop and the closed, folded triple loop. We adjusted the initial, or "natural" curvature by first cutting the loop, and then passing it through some mechanical rollers, which caused plastic curving about the cross-sectional width. The ends of cut loop are then re-connected by a simple clasp, visible here, before folding into higher-order configurations (see Fig. 2). Subfigures (a)-(c) show stable states with one, three and five loops (top and bottom views); in (c') the five loop case has partially unraveled but is locked by frictional interplay between the loops. In (d), the naturally curved rod for each of these cases - the ends are free and it has just over three turns. 

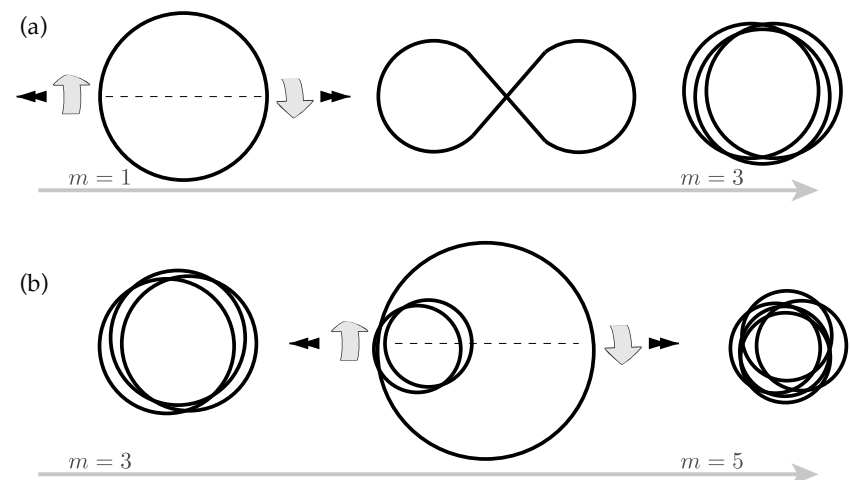

Figure 2: Folding sequence for a loop with one, three and five stable loop configurations. (a) A single loop is twisted along a given diameter by hand to form a figure-of-eight loop in transition to the final three loop state. (b) Folding three loops into five is accomplished by, first, manually feeding arc-length into one of the three loops, at the expense of the other two, and then twisting this larger loop as per the single loop. The resulting five-looped state then adjusts itself automatically to the same radius everywhere even though we show them as being slightly different-purely to convey the number of loops.

loops is increased by feeding the arc-length of the two other loops into this larger loop by hand: when the larger loop is around 2-3 times as wide, it can be twisted in a manner similar to the single loop, which forms three more loops in addition to the current pair of smaller loops. Once released, the final five loops adopt the same radii. Folding five- into seven loops is performed similarly provided plastic strains are not incurred. Each shape, or state, has different degrees of proximal stability: by far, the three loop configuration is most stable, and the other states move towards it in sequence if significantly perturbed or deliberately unfolded; from the single loop to three, or from seven- to five- to three loops. This happens to be the case for this physical model; if the intrinsic curvature is different, the stability character is altered.

A simple experiment is then performed to assess the limits at which stability is lost. For a given $\kappa_{\mathrm{n}}$, the length of the blade is varied by feeding it through the bracket, thereby altering the closed length and $r_{0}$. The set-up is then laid on a horizontal table and gently released; stability is defined as being lost when the configuration either folds into more turns or opens up into fewer turns, and the critical data are reported in table 1. In particular, it is found that a single loop is stable only if $r_{0} \kappa_{\mathrm{n}}$ is less than 3.5 . Above this value, a single loop cannot exist in an unloaded state but spontaneously collapses into three loops. The example in Fig. 1 has a ratio $r_{0} \kappa_{\mathrm{n}}$ just below this value, and so is stable, but the degree of perturbation needed for destabilizing this shape is small. Three loops are just stable if $r_{0} \kappa_{\mathrm{n}}$ is larger than 0.28 , otherwise they open into a single loop for smaller values of this ratio. If the ratio is increased, then the system is 
Table 1: Experimental regions of stability expressed in terms of $r_{0} \kappa_{\mathrm{n}}$ (current radius $\times$ natural, open curvature) using a steel bandsaw blade, of rectangular cross-section $5.65 \mathrm{~mm} \times 0.65 \mathrm{~mm}$ and $L \approx 3 \mathrm{~m}$.

\begin{tabular}{cc}
\hline covering index $m$ & stability bounds \\
\hline 1 & $r_{0} \kappa_{\mathrm{n}}<3.50$ \\
3 & $0.28<r_{0} \kappa_{\mathrm{n}}<$ [beyond elastic limit] \\
5 & $0.42<r_{0} \kappa_{\mathrm{n}}<$ [beyond elastic limit]
\end{tabular}

increasingly resistant to perturbations. Unlike a single loop, three loops unfold, and the same is true for five loops, which open up into three loops if $r_{0} \kappa_{\mathrm{n}}<0.42$.

Other limits were considered, for example, for seven loops unfolding into five, but this was not recorded because there was too much contact between the loops, and friction played a significant role in preventing the transition. We also surmised that each of the three- and five loop scenarios were governed by an upper bound on stability in terms of $r_{0} \kappa_{\mathrm{n}}$, where a higher ratio would cause each case to be unstable in the direction of more loops, i.e. three loops would spontaneously collapse into five loops etc. However, this was not observable without incurring plastic deformation because the original loop radius needs to be very small to unfold into a radius at least five-to-tenfold larger. In addition, higher loop configurations are reached only by folding in a very precise way, which is unlikely to happen spontaneously.

\section{Models: elastic ribbon versus elastic rods}

In this section, we introduce three different mechanical models that can be used to represent a naturally curved elastic strip, such as the previously discussed bandsaw blade. The ribbon model is the only one that can accurately capture situations where the thickness $h$, the cross-sectional width $w$ and the curvilinear length $L$ are all very different, with $h \ll w \ll L$ : this model is derived from a two-dimensional inextensible plate model by considering the limit $w \ll L$. The two other models are given merely for the sake of comparison; they are less accurate, but are somewhat simpler and have been extensively used in prior work. One model is the isotropic rod model: this is the standard model for a thin elastic rod having a circular cross-section - it simply ignores the aspect-ratio of the cross-section. The other one is the flat rod model, obtained by extrapolating the thin elastic rod model for a rectangular cross-section with dimensions $w \times h$ to the limit of a flat cross-section, i.e. by letting $h / w \rightarrow 0$. This limit $h / w \rightarrow 0$, however, is incompatible with Kirchhoff kinematical hypothesis, which underpins the derivation of all rod models. The flat rod model is therefore mathematically defined, but ill-justified from a mechanical perspective. 


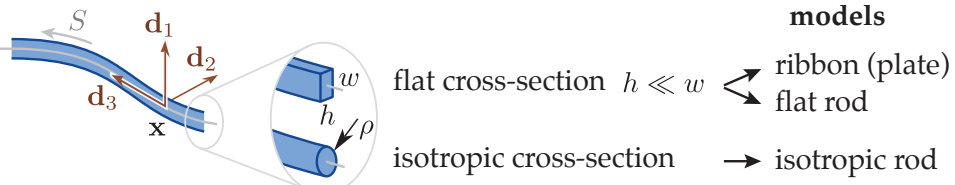

Figure 3: An elastic ribbon is represented as a Cosserat curve $\mathbf{x}(S)$ with directors $\mathbf{d}_{i}(S)$. Throughout this paper, we compare the predictions of three mechanical models based on this kinematical description: the accurate ribbon model (which extends Sadowsky's narrow plate model to account for natural curvature), the classical rod model for a rectangular cross-section in the limit where the latter has a small aspect ratio, $h \ll w$ (flat rod model), and for a circular cross-section (isotropic rod model).

\subsection{Inextensible Cosserat curve}

We start with a kinematical description, common to all three models. Let $S$ denote the arc-length in a reference configuration, and let $L$ denote the curvilinear length of the rod. We use $S \in[0, L]$ as a Lagrangian coordinate to follow, or track, the location of material cross-sections. We will mostly consider static problems. However, to analyze dynamic stability, we will occasionally introduce a time variable, which we denote by $\tau$. For any function $f(S, \tau)$, the derivative with respect to position in the reference configuration is denoted by $f^{\prime}(S, \tau)=\partial f / \partial S$ and the temporal derivative by $\dot{f}(S, \tau)=\partial f / \partial \tau$.

Vectors are denoted by boldface characters. A configuration is specified by a Cosserat curve, i.e. by the centerline $\mathbf{x}(S)$ and by three orthonormal directors $\mathbf{d}_{i}(S)$, with $i=1,2,3$. We consider only inextensible and unshearable materials, for which the following two kinematical constraints are applicable. First, one of the directors, which we choose to be $\mathbf{d}_{3}$, coincides with the tangent to the curve:

$$
\mathbf{x}^{\prime}(S)=\mathbf{d}_{3}(S)
$$

Second, the director's frame is orthonormal,

$$
\mathbf{d}_{i}(S) \cdot \mathbf{d}_{j}(S)=\delta_{i j},
$$

which must hold for any $0 \leq S \leq L$ and for any combination of indices $(i, j)$, where $\delta_{i j}$ denotes Kronecker's symbol. By convention, $\mathbf{d}_{1}$ is along the width of the strip and $\mathbf{d}_{2}$ is perpendicular to the plane tangent to strip, see figure 3 . Note that inextensibility follows from projecting equation (1) along the tangent direction $\mathbf{d}_{3}$ : this yields $\left|\mathbf{x}^{\prime}\right|=1$, i.e. $|\mathrm{d} \mathbf{x}|=|\mathrm{d} S|$.

For closed ribbons, the centerline and directors are periodic, such that $\mathbf{x}(L)=\mathbf{x}(0)$ and $\mathbf{d}_{i}(L)=\mathbf{d}_{i}(0)$ (the equilibrium requires that the internal stress is period as well, and this will be imposed later).

The curvature strains $\left(\omega_{1}, \omega_{2}\right)$ and the twisting strain $\omega_{3}$ are defined as the components of the rotation gradient $\boldsymbol{\omega}$ relative to the basis formed by the 
directors:

$$
\omega_{i}(S)=\frac{1}{2} \sum_{j=1}^{3} \sum_{k=1}^{3} \epsilon_{i j k} \mathbf{d}_{j}^{\prime}(S) \cdot \mathbf{d}_{k}(S)
$$

where $\epsilon_{i j k}$ is the alternating symbol. The rotation gradient, also known as the "Darboux vector", is represented by $\boldsymbol{\omega}=\sum_{i=1}^{3} \omega_{i} \mathbf{d}_{i}$ and obeys $\mathbf{d}_{i}^{\prime}=\boldsymbol{\omega} \times \mathbf{d}_{i}$.

\subsection{Kirchhoff's equations of motion}

To state the equations of motion for ribbons and rods, we introduce the time variable $\tau$. The internal stress is represented by the resultant $\mathbf{R}(S, \tau)$ and by the moment $\mathbf{M}(S, \tau)$. In the absence of external forces, the linear and angular momentum balances are expressed by Kirchhoff's equations of motion,

$$
\begin{aligned}
\mathbf{R}^{\prime}(S, \tau) & =\rho \ddot{\mathbf{x}}(S, \tau) \\
\mathbf{M}^{\prime}(S, \tau)+\mathbf{x}^{\prime}(S, \tau) \times \mathbf{R}(S, \tau) & =\mathbf{0},
\end{aligned}
$$

where $\rho$ is the mass per unit length. Since we have ignored the rotational inertia of the cross-section, the right-hand side of the equation for moment equilibrium is zero; as we explain later, this has implications for the growth rate of twistingmode instabilities.

\subsection{Constitutive laws}

The components $M_{i}=\mathbf{M} \cdot \mathbf{d}_{i}$ of the internal moment $\mathbf{M}$ relative to the basis of the directors are given by constitutive laws in terms of the strains $\omega_{i}$. The three mechanical models (ribbon, isotropic rod, flat rod) arise from different constitutive laws, all of which are denoted generically by $\mathbf{f}=\mathbf{0}$,

$$
\mathbf{f}\left(M_{1}, M_{2}, M_{3}, \omega_{1}, \omega_{2}, \omega_{3}\right)=\mathbf{0} .
$$

Expressions for $\mathbf{f}$ corresponding to the different models are proposed below.

\subsubsection{Constitutive law for a ribbon}

The ribbon model was introduced in the seminal work of Sadowsky 20, 21, 22. He derived the energy of an elastic ribbon by dimensional reduction by starting from the energy of a narrow, two-dimensional elastic plate, which he reduced to a one-dimensional model using the developability condition. His model correctly captures the inextensibility of the midsurface of the ribbon, which therefore remains a developable (hence ruled) surface after deformation ${ }^{2}$ Sadowsky explicitly parameterizes the ribbon's midsurface using the inverse cotangent $\eta$ of the angle between the generatrices and the tangent $\mathbf{d}_{3}$ to the centerline.

\footnotetext{
${ }^{2}$ By contrast, Kirchhoff's kinematical hypothesis, which underpins rod models, valid when $w$ and $h$ are comparable, but it does not capture developable configurations well in the limit $h \ll w$.
} 
Using a variational method, Starostin and van der Heijden 22] derived the equilibrium equations found by Sadowsky [18, 11, 20, 9, which they applied to geometries such as that in 23. Even though their variational method departs from the standard treatment of thin elastic rods, the equilibrium equations they obtain are very similar to the classical Kirchhoff equations for thin rods, as they note.

In another paper in this special issue [3], Dias and Audoly extend Sadowsky's model to handle natural curvature. They also derive equilibrium equations for ribbons by adapting the classical approach, i.e. by writing a principle of virtual work for elastic ribbons analogous to elastic rods. This viewpoint makes evident the strong similarities between the governing equations for rods and ribbons. It it also has a practical advantage: it makes ribbons appear as a special case of rods. We adopt this view in the present paper, which enables us to analyze the stability of elastic ribbons by adapting of the classical stability analyses for elastic rods in a straightforward way.

We temporarily drop the time variable $\tau$, as it does not enter into the constitutive laws. The direction of generatrices is parameterized by the quantity $\eta(S)$ in the Sadowsky model, and developability implies the constraint

$$
\omega_{3}(S)=\eta(S) \omega_{1}(S) .
$$

Following [3], we refrain from eliminating the parameter $\eta$, and view instead $\eta$ and $\omega_{3}$ as two variables linked by a kinematical constraint. The ribbons we consider here have zero geodesic curvature, i.e. $\omega_{2}=0$ in the reference configuration, and their midsurface is inextensible, as previously noted. The geodesic curvature is preserved upon isometric deformations of the midsurface, hence the kinematical constraint

$$
\omega_{2}=0
$$

persists during deformation.

As written in Section 2, $\kappa_{\mathrm{n}}$ denotes the natural out-of-plane curvature. For simplicity, we assume $\kappa_{\mathrm{n}}$ is constant, and the reference configuration is taken to be a section of open cylinder with height $w$ and natural radius, $\kappa_{\mathrm{n}}{ }^{-1}$. The strain energy stored in the deformed ribbon is derived in equation (34) of reference [3] from the energy of an inextensible cylindrical shell as

$$
\mathcal{E}_{\mathrm{rib}}=\frac{B_{\mathrm{rib}}}{2} \int_{0}^{L}\left[\omega_{1}^{2}\left(1+\eta^{2}\right)^{2}-2 \kappa_{\mathrm{n}} \omega_{1}\left(1+\nu \eta^{2}\right)\right] \mathrm{d} S,
$$

where $B_{\text {rib }}=D w$, is the product of the plate modulus $D=\frac{E h^{3}}{12\left(1-\nu^{2}\right)}$ and the width $w$ of the ribbon; $E$ is Young's modulus and $\nu$ is Poisson's ratio. The first term inside parenthesis in the integrand is Sadowsky's energy, and the second term captures the effect of the natural curvature $\kappa_{\mathrm{n}}$.

The first variation of the energy $\mathcal{E}_{\text {rib }}$ enables the following constitutive law to be found when the constraints in equations $6 \mathrm{a}$ and $6 \mathrm{~b}$ are applied:

$$
M_{1}=\frac{\partial \mathcal{E}_{\text {rib }}}{\partial \omega_{1}}-\eta M_{3}
$$


Moreover, the condition of equilibrium for $\eta$ writes:

$$
\frac{\partial \mathcal{E}_{\text {rib }}}{\partial \eta}-\omega_{1} M_{3}=0
$$

We refer the reader to $\left[3\right.$ for details. Note that $M_{3}$ is the Lagrange multiplier associated with the constraint 6a): the principle of virtual work for ribbons is a constrained variational problem, and the terms $\left(-\eta M_{3}\right)$ and $\left(-\omega_{1} M_{3}\right)$ in equations $8 \mathrm{a}$ and $8 \mathrm{~b}$ are constraint terms.

Using equation (8b), we have $M_{3}=2 B_{\text {rib }}\left(\omega_{1}\left(1+\eta^{2}\right) \eta-\kappa_{\mathrm{n}} \nu \eta\right)$; equation (8a) then yields $M_{1}=B_{\text {rib }}\left(\omega_{1}\left(1-\eta^{4}\right)-\kappa_{\mathrm{n}}\left(1-\nu \eta^{2}\right)\right)$. Eliminating $\eta$ from equation (6a), the two constitutive equations and the constitutive constraint for a naturally curved ribbon are expressed altogether by $\mathbf{f}_{\text {rib }}=0$, with

$$
\mathbf{f}_{\text {rib }}=\left(\begin{array}{c}
M_{1}-B_{\text {rib }}\left[\omega_{1}-\frac{\omega_{3}^{4}}{\omega_{1}^{3}}-\kappa_{\mathrm{n}}\left(1-\nu \frac{\omega_{3}^{2}}{\omega_{1}^{2}}\right)\right] \\
\omega_{2} \\
M_{3}-2 B_{\text {rib }}\left[\omega_{3}+\frac{\omega_{3}^{3}}{\omega_{1}{ }^{2}}-\kappa_{\mathrm{n}} \nu \frac{\omega_{3}}{\omega_{1}}\right]
\end{array}\right)
$$

Only the first and third components of $\mathbf{f}_{\text {rib }}=\mathbf{0}$ are genuine constitutive laws; the second component yields a constitutive constraint $\omega_{2}=0$, which is the geodesic constraint introduced earlier in equation (6b): in the presence of a kinematical constraint affecting a strain measure, $\omega_{2}=0$, this constraint indeed replaces the constitutive law for $\omega_{2}$ and the bending moment $M_{2}$ is determined by solving the full equilibrium problem.

The ribbon model is fully captured by the expression of $\mathbf{f}_{\text {rib }}$ proposed in equation (9), so we do not need to use equations $(6-8)$ henceforth. It has been checked in reference [3] that the equations of equilibrium (4) and the constitutive law $\mathbf{f}_{\text {rib }}=\mathbf{0}$ are equivalent to the equilibrium equations derived by Starostin and van der Heijden 22, in the special case of a naturally straight and static ribbon $\left(\kappa_{\mathrm{n}}=0\right.$ and $\left.\ddot{\mathbf{x}}=\mathbf{0}\right)$.

\subsection{Constitutive law for an isotropic rod}

We define an 'isotropic' rod to be a linear elastic rod having equal principal bending moduli, as for a circular cross-section (note that isotropic rods may be naturally curved). The classical rod model representing an isotropic rod with natural curvature $\kappa_{\mathrm{n}}$ expresses $\mathbf{f}_{\text {iso }}$ as

$$
\mathbf{f}_{\text {iso }}=\left(\begin{array}{c}
M_{1}-B_{\text {iso }}\left(\omega_{1}-\kappa_{\mathrm{n}}\right) \\
M_{2}-B_{\text {iso }} \omega_{2} \\
M_{3}-C_{\text {iso }} \omega_{3} .
\end{array}\right)
$$

In its natural configuration, a section of this rod is a torus with cross-sectional radius $\rho$, enclosing a circular centerline with radius $\kappa_{\mathrm{n}}{ }^{-1}$. The bending and twisting moduli read $B_{\text {iso }}=E \frac{\pi \rho^{4}}{4}, C_{\text {iso }}=\frac{E}{2(1+\nu)} \frac{\pi \rho^{4}}{2}$, respectively, where $\mu=\frac{E}{2(1+\nu)}$ is the shear modulus of an isotropic Hookean material. 


\subsection{Constitutive law for a flat rod}

We call 'flat rod' the mathematical limit $w \gg h$ of the rod model with a rectangular cross-section, $w \times h$, and a natural curvature $\kappa_{\mathrm{n}}$. When the aspect-ratio, $h / w$, of the cross-section tends to zero, the constitutive law is obtained in the limit:

$$
\mathbf{f}_{\text {flat }}=\left(\begin{array}{c}
M_{1}-B_{\text {flat }}\left(\omega_{1}-\kappa_{\mathrm{n}}\right) \\
\omega_{2} \\
M_{3}-C_{\text {flat }} \omega_{3}
\end{array}\right)
$$

where $B_{\text {flat }}=E \frac{w h^{3}}{12}, C_{\text {flat }}=\frac{E}{2(1+\nu)} \frac{w h^{3}}{3}$. Note that $B_{\text {flat }}$ and $C_{\text {flat }}$ are of the same order of magnitude, $\sim w h^{3}$. The other principal bending modulus is of order $\sim w^{3} h$, and is therefore much larger: this is why we replaced the corresponding constitutive law with the kinematical constraint $\omega_{2}=0$. For a rectangular rod, the torsional constant is obtained by solving a Laplace equation in a rectangular domain. The value of the torsional constant $\frac{w h^{3}}{3}$ used here corresponds to the solution of this Laplace problem for a thin strip of dimensions $w \times h$.

\subsection{Not all models were created equal}

As discussed at the beginning of $\$ 3$, the ribbon model is the only accurate model if $h \ll w \ll L$. The two other models cannot be justified asymptotically in this limit and, worse, they are based on kinematical assumptions that cannot capture the inextensible modes of deformation of the center-surface (which are the lowest-energy modes when $h \ll w$ ). The rod models are given only for the sake of comparison.

\section{Linearized equations of motion near circular solutions}

Without specifying boundary conditions, we linearize these equations near a circular configuration, as shown in figure 4. In so doing, we obtain the tangent bending and twisting moduli associated with the different constitutive laws, which play a key role in the stability analysis of the ribbon. The general linearized equations of motion derived in the present section are applied later to two specific geometries in $\$ 5$ and $\$$

\subsection{Base state: circular solutions}

We consider the twistless, circular state shown in figure 4 and analyze its stability for different boundary conditions. All quantities pertaining to the base circular state are marked with a subscript or superscript ' 0 '. Let therefore $\omega_{1}^{0}$ denote the curvature in the circular state, and $r_{0}=1 / \omega_{1}^{0}$ the radius of curvature, as in figure 4. The centerline is parameterized by $\mathbf{x}_{0}(S)=r_{0} \mathbf{e}_{r}(\theta)$ where $\theta=\omega_{1}^{0} S$ is the polar angle. The directors read $\mathbf{d}_{1}^{0}(S)=\mathbf{e}_{z}, \mathbf{d}_{2}^{0}(S)=\mathbf{e}_{r}(\theta), \mathbf{d}_{3}^{0}(S)=\mathbf{e}_{\theta}(\theta)$. 


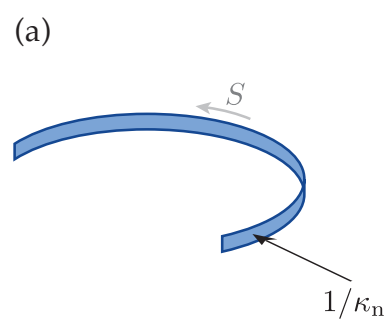

(b)

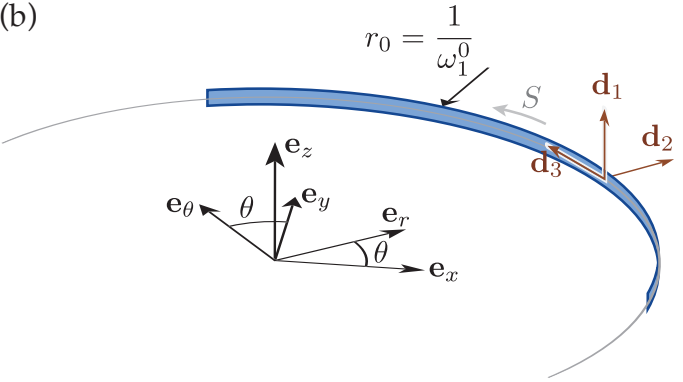

Figure 4: (a) Reference configuration of a ribbon with natural curvature $\kappa_{\mathrm{n}}$. (b) A circular actual configuration with curvature $\omega_{1}^{0}=1 / r_{0}$.

The kinematical constraints in equations $\sqrt{1-2}$ are satisfied, as can be checked. The rotation gradient $\boldsymbol{\omega}^{0}=\omega_{1}^{0} \mathbf{e}_{z}$ has components $\left(\omega_{1}^{0}, \omega_{2}^{0}, \omega_{3}^{0}\right)=\left(\omega_{1}^{0}, 0,0\right)$ in the directors basis $\left(\mathbf{d}_{1}^{0}, \mathbf{d}_{2}^{0}, \mathbf{d}_{3}^{0}\right)$.

In the present setting, the twisting strain is zero $\omega_{3}=0$. Observe that the three constitutive laws all predict similar expressions for the bending and twisting moment, $M_{1}^{0}=B\left(\omega_{1}^{0}-\kappa_{\mathrm{n}}\right)$ and $M_{3}^{0}=0$ when $\omega_{3}=0$. Here $B$ denotes the bending modulus relevant to the model under consideration, $B=B_{\text {rib }}$, $B_{\text {iso }}$ or $B_{\text {flat }}$. Now, let us calculate $M_{2}^{0}$. For the isotropic rod model, $M_{2}^{0}=0$ follows directly from the constitutive law. For the two other models, $M_{2}^{0}=0$ follows from equilibrium: the balance of moments in equation $4 \mathrm{~b}$ implies $0=$ $\left(\mathbf{M}_{0}^{\prime}+\mathbf{d}_{3}^{0} \times \mathbf{R}_{0}\right) \cdot \mathbf{d}_{3}^{0}=\mathbf{M}_{0}^{\prime} \cdot \mathbf{d}_{3}^{0}=M_{3}^{0^{\prime}}+\omega_{1}^{0} M_{2}^{0}=\omega_{1}^{0} M_{2}^{0}$. Therefore, in all cases we have

$$
\mathbf{M}_{0}(S)=M_{1}^{0} \mathbf{d}_{1}^{0}, \quad \text { where } M_{1}^{0}=B\left(\omega_{1}^{0}-\kappa_{\mathrm{n}}\right) .
$$

The consequence of equation $12 \mathrm{a}$ is that $\mathbf{M}_{0}^{\prime}=\mathbf{0}$. Projecting now the balance of moments $\mathbf{M}_{0}^{\prime}+\mathbf{d}_{3}^{0} \times \mathbf{R}_{0}=\mathbf{0}$ onto $\mathbf{d}_{1}^{0}$ and $\mathbf{d}_{2}^{0}$, we find that $R_{1}^{0}=R_{2}^{0}=0$. The balance of forces is given by $(4 \mathrm{a})$ with $\ddot{\mathbf{x}}=\mathbf{0}$ : this yields $\left(R_{3}^{0} \mathbf{d}_{3}^{0}\right)^{\prime}=\mathbf{0}$; hence $R_{3}^{0}=0$ and so

$$
\mathbf{R}_{0}(S)=\mathbf{0} .
$$

By 12 , the pre-stress in a circular configuration is a pure bending moment: $\left(\mathbf{R}_{0}(S), \mathbf{M}_{0}(S)\right)=\left(\mathbf{0}, M_{1}^{0} \mathbf{d}_{1}^{0}\right)$. This bending moment drives the instabilities which we analyze later. The opposite case of instabilities driven by a twisting moment has been studied in the classical paper of Michell [16], see also [5].

\subsection{Mode of vibration near a circular configuration}

We consider a vibration mode near this circular configuration, i.e. assume a small perturbation away from the circular state depending harmonically on the time variable $\tau$. We use the notation of complex analysis and denote by $\hat{\mathbf{x}}$ the complex amplitude of displacement, by $\hat{\boldsymbol{\psi}}$ the complex infinitesimal rotation vector, by $\hat{\mathbf{R}}$ the complex amplitude of the internal force perturbation, and by 
$\hat{\mathbf{M}}$ the complex amplitude of the internal moment:

$$
\begin{aligned}
\mathbf{x}(S, t) & =\mathbf{x}_{0}(S)+\left[r_{0}\right] \hat{\mathbf{x}}(\bar{S}) e^{i \Omega \tau} \\
\mathbf{d}_{i}(S, t) & =\mathbf{d}_{i}^{0}(S)+\left(\hat{\boldsymbol{\psi}}(\bar{S}) e^{i \Omega \tau}\right) \times \mathbf{d}_{i}^{0}(S) \\
\mathbf{R}(S, t) & =\mathbf{0}+\left[B\left(\omega_{1}^{0}\right)^{2}\right] \hat{\mathbf{R}}(\bar{S}) e^{i \Omega \tau} \\
\mathbf{M}(S, t) & =\mathbf{M}_{0}+\left[B \omega_{1}^{0}\right] \hat{\mathbf{M}}(\bar{S}) e^{i \Omega \tau}
\end{aligned}
$$

The perturbations $(\hat{\mathbf{x}}, \hat{\boldsymbol{\psi}}, \hat{\mathbf{R}}, \hat{\mathbf{M}})$ have been nondimensionalized by including the appropriate scaling factors in square brackets on the right-hand side. Our dimensionless units make use of the reference length $r_{0}=1 / \omega_{1}^{0}$ (radius of the center-line the circular base state) and of the reference moment $B \omega_{1}^{0}$. All other reference quantities, such as forces and energies, are obtained by combining $r_{0}$ and $B \omega_{1}^{0}$. In equation $(13)$, the perturbations $(\hat{\mathbf{x}}, \hat{\boldsymbol{\psi}}, \hat{\mathbf{R}}, \hat{\mathbf{M}})$ are sought as a function of the rescaled arc-length defined by

$$
\bar{S}=\omega_{1}^{0} S .
$$

Note that the expression for the director $\mathbf{d}_{i}$ in equation $13 \mathrm{~b}$ in terms of the infinitesimal rotation $\hat{\boldsymbol{\psi}}$, automatically preserves the orthonormality between directors to a linear order in the perturbation.

\subsection{Linearized constitutive law}

The constitutive law has been written in a generic form as $\mathbf{f}=0$ in equation (5), and special forms relevant to the different models have been obtained in equations (9), 10) and (11). We now linearize these constitutive laws near the circular configuration

$$
\left(M_{1}, M_{2}, M_{3}, \omega_{1}, \omega_{2}, \omega_{3}\right)=\left(M_{1}^{0}, 0,0, \omega_{1}^{0}, 0,0\right) .
$$

In terms of the increments of strain $\tilde{\omega}_{i}$ and increments of bending and twisting moments $\tilde{M}_{i}$, the linearized constitutive laws all take the form

$$
\begin{aligned}
& \tilde{M}_{1}=B \tilde{\omega}_{1} \\
& \tilde{M}_{2}=B g \tilde{\omega}_{2} \\
& \tilde{M}_{3}=B t \tilde{\omega}_{3},
\end{aligned}
$$

where $B$ is the model-dependent principal bending modulus introduced earlier, and $(t, g)$ are the dimensionless second bending modulus and dimensionless twisting modulus, respectively. Both $t$ and $g$ are model-dependent as well: their expressions are listed in table 2. The tangent moduli $t$ and $g$ have a strong influence on the stability of circular configurations: in particular, the tangent twisting moduli $g$ predicted by the ribbon and flat rod models have different expression and this implies, as we shall confirm later, that these two models have different stability properties. 
Table 2: Values of the dimensionless tangent elastic moduli predicted by the different models near a circular configuration with radius $r_{0}$. These coefficients are obtained by linearizing equations (9 11 ) and identifying the result with the form proposed in equation (15).

contrast of bending moduli $g$ dimensionless twisting modulus $t$

\begin{tabular}{rcc}
\hline ribbon & $\infty$ & $2\left(1-\nu \kappa_{\mathrm{n}} r_{0}\right)$ \\
flat rod & $\infty$ & $\frac{2}{1+\nu}$ \\
isotropic rod & 1 & $\frac{1}{1+\nu}$ \\
\hline
\end{tabular}

In table 2, the statement of $g=\infty$ for the ribbon and flat rod models suggests that equation (15b) should then be replaced by $\tilde{\omega}_{2}=0$ (which is indeed the incremental form of the kinematical constraint $\omega_{2}=0$ ).

Note that the increments $\tilde{M}_{i}$ of the bending and twisting moments $M_{i}=$ $\mathbf{M} \cdot \mathbf{d}_{i}$ differ from the components $\hat{M}_{i}=\hat{\mathbf{M}} \cdot \mathbf{d}_{i}^{0}$ of the perturbed internal moment vector, $\hat{\mathbf{M}}$, onto the unperturbed directors basis: $\tilde{M}_{i}$ should not be confused with $\hat{M}_{i}$. For instance, for an infinitesimal rigid-body rotation, $\hat{\mathbf{M}}$ follows the rotation and its components $\hat{M}_{i}$ in the unperturbed directors basis are non-zero, although the quantities $M_{i}$ are constant, so $\tilde{M}_{i}=0$. Likewise, $\tilde{\omega}_{i}$ denote the increments of the strains $\omega_{i}$ and not the components of a vector $\hat{\boldsymbol{\omega}}$ (they are in fact the components of $\hat{\boldsymbol{\psi}}^{\prime}$, as we note later).

\subsection{Equations for the modes}

Linearizing the kinematical condition (1), the equations of motion (4), the definition of twist-curvature strains in equation (3) and using the linearized constitutive law in equation (15), we obtain

$$
\begin{gathered}
\hat{\mathbf{x}}^{\prime}(\bar{S})=\hat{\boldsymbol{\psi}} \times \mathbf{d}_{3}^{0}, \\
\hat{\mathbf{R}}^{\prime}(\bar{S})=-\bar{\Omega}^{2} \hat{\mathbf{x}}, \\
\hat{\mathbf{M}}^{\prime}(\bar{S})+\mathbf{d}_{3}^{0} \times \hat{\mathbf{R}}=\mathbf{0}, \\
\hat{\mathbf{M}}=\hat{\boldsymbol{\psi}} \times \overline{\mathbf{M}}_{0}+\left(\mathbf{d}_{1}^{0} \otimes \mathbf{d}_{1}^{0}+g \mathbf{d}_{2}^{0} \otimes \mathbf{d}_{2}^{0}+t \mathbf{d}_{3}^{0} \otimes \mathbf{d}_{3}^{0}\right) \cdot \hat{\boldsymbol{\psi}}^{\prime}(\bar{S}),
\end{gathered}
$$

where we have defined the dimensionless pre-stress $\overline{\mathbf{M}}_{0}=\bar{M}_{1}^{0} \mathbf{d}_{1}^{0}$ (which is identical for all models) and the dimensionless angular velocity $\bar{\Omega}$ by

$$
\begin{aligned}
\bar{M}_{1}^{0} & =\frac{M_{1}^{0}}{B \omega_{1}^{0}}=1-\kappa_{\mathrm{n}} r_{0}, \\
\bar{\Omega} & =\frac{\Omega}{\sqrt{\frac{B}{\rho}}\left(\omega_{1}^{0}\right)^{2}} .
\end{aligned}
$$


Equation 16d can be interpreted by observing ${ }^{3}$ that $\hat{\mathbf{M}}-\hat{\boldsymbol{\psi}} \times \overline{\mathbf{M}}_{0}=$ $\sum_{i} \tilde{M}_{i} \mathbf{d}_{i}^{0}$, and that $\hat{\boldsymbol{\psi}}^{\prime}=\sum_{i} \tilde{\omega}_{i} \mathbf{d}_{i}^{0}$. When projected along any one of the undeformed directors $\mathbf{d}_{i}^{0}$, equation $16 \mathrm{~d}$ ) then appears to be equivalent to any one of the linearized constitutive laws in equation (8), in nondimensional form, i.e. with $B$ set to 1 .

To solve these equations, it will be convenient to represent the unknown amplitudes relative to the unperturbed director basis:

$$
\begin{aligned}
\hat{\mathbf{x}}(\bar{S}) & =\sum_{i=1}^{3} \hat{x}_{i}(\bar{S}) \mathbf{d}_{i}^{0}(\bar{S}), & \hat{\psi}(\bar{S}) & =\sum_{i=1}^{3} \hat{\psi}_{i}(\bar{S}) \mathbf{d}_{i}^{0}(\bar{S}), \\
\hat{\mathbf{R}}(\bar{S}) & =\sum_{i=1}^{3} \hat{R}_{i}(\bar{S}) \mathbf{d}_{i}^{0}(\bar{S}), & \hat{M}(\bar{S}) & =\sum_{i=1}^{3} \hat{M}_{i}(\bar{S}) \mathbf{d}_{i}^{0}(\bar{S}) .
\end{aligned}
$$

In dimensionless units, the unperturbed rotation gradient $\boldsymbol{\omega}_{1}^{0}=\omega_{1}^{0} \mathbf{e}_{z}$ reads simply as $\overline{\boldsymbol{\omega}}_{1}^{0}=\mathbf{e}_{z}$. Therefore, any one of the amplitudes $(\hat{\mathbf{x}}, \hat{\boldsymbol{\psi}}, \hat{\mathbf{R}}, \hat{\mathbf{M}})$, denoted generically by $\hat{\mathbf{v}}(\bar{S})$, is differentiated in the moving basis according to the formula

$$
\hat{\mathbf{v}}^{\prime}(\bar{S})=\mathbf{e}_{z} \times \hat{\mathbf{v}}+\sum_{i=1}^{3} \hat{v}_{i}^{\prime} \mathbf{d}_{i}^{0}=\hat{v}_{1}^{\prime} \mathbf{d}_{1}^{0}+\left(\hat{v}_{2}^{\prime}-\hat{v}_{3}\right) \mathbf{d}_{2}^{0}+\left(\hat{v}_{3}^{\prime}+\hat{v}_{2}\right) \mathbf{d}_{3}^{0} .
$$

In the forthcoming sections, we analyze the stability of rods and ribbons in different circular geometries: we consider the unfolding of a semicircular ribbon in $\$ 5$ and the stability of $m$-covered circles in $\$ 6$. These stability analyses make use of the equations derived above: we insert the decompositions of the perturbations into components given in equation (18) into the linearized equations of motion (16), making use of the differentiation rule in equation (19). In so doing, we obtain a set of twelve coupled linear ordinary differential equations - which we supplement by appropriate boundary conditions.

\section{Unfolding of a semicircular ribbon}

Our analysis of the linearized constitutive laws has revealed a distinctive feature of the ribbon model: its tangent twisting modulus $t_{\text {rib }}=2\left(1-\nu \kappa_{\mathrm{n}} r_{0}\right)$ in dimensionless form becomes negative when the curvature reaches the value $r_{0}^{-1}=\nu \kappa_{\mathrm{n}}$. This suggests that a ribbon can become unstable when it is unbent, or undercurved, i.e. when the curvature is decreased from its natural value $\kappa_{\mathrm{n}}$. In this section, we consider the simplest geometry for which such an instability can appear: the unfolding of a semicircular strip. A linear stability analysis is first carried out, and predictions from the ribbon model are contrasted with those of the rod models, whose tangent twisting modulus is always positive, irrespective of the value of $r_{0}^{-1}$. A finite element analysis of a shell model is conducted, which confirms our findings.

\footnotetext{
${ }^{3}$ To show these equalities, note that the left-hand side of the first equation is the derivative of $\hat{\mathbf{M}}$ in the moving basis following the virtual motion. The second equation is a classical formula for calculating the bending and twisting strain increments; see for instance [2].
} 


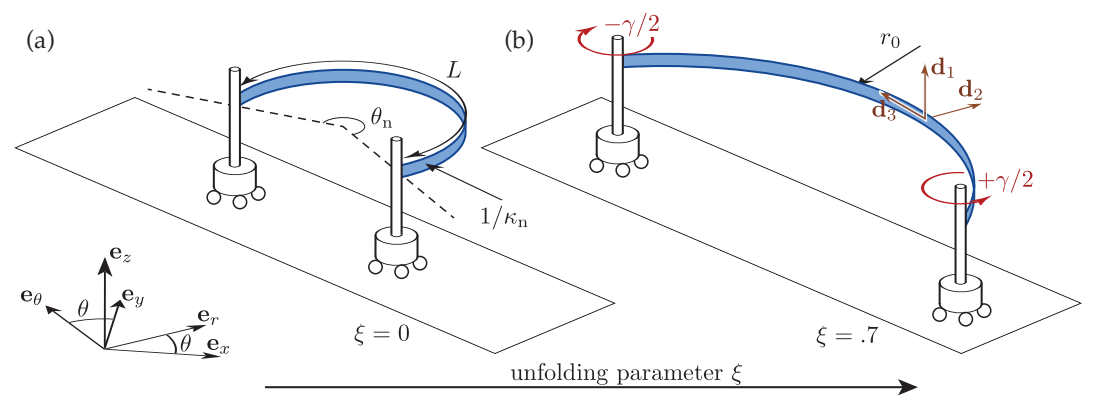

Figure 5: Unfolding a semicircular ribbon. (a) Natural configuration: the case of a semicircle corresponds to $\theta_{\mathrm{n}}=\pi$. (b) Undercurved, circular configuration with differential rotation of the ends $\gamma=\theta_{\mathrm{n}} \xi$; its stability is analyzed in $\$ 5.3$.

\subsection{Geometry}

We consider a naturally curved ribbon of arclength $L$, subtending an initial angle $\theta_{\mathrm{n}}=L \kappa_{\mathrm{n}}$. It is progressively flattened by applying equal and opposite rotations to both ends normal to the $(O x y)$ plane; see figure 5 . Out-of-plane displacements are completely restrained, but the ends are free to move without friction towards, or away from, each other in-plane. The end-wise rotations take place about the transverse direction $\mathbf{e}_{z}$ and the relative rotation, $\gamma$, of the two ends is expressed in terms of an unfolding parameter $\xi$ as $\gamma=\theta_{\mathrm{n}} \xi$; the initial, natural configuration corresponds to $\xi=0$, and the flattened configuration corresponds to $\xi=1$. We analyze the stability of the circular configuration during unfolding, i.e. as $\xi \geq 0$ is increased.

\subsection{Base circular solution}

There exists a planar, circular solution to this problem, with curvature $\omega_{1}^{0}=$ $\kappa_{\mathrm{n}}(1-\xi)$, hence a radius of curvature $r_{0}=\left(\omega_{1}^{0}\right)^{-1}=\frac{\kappa_{\mathrm{n}}^{-1}}{(1-\xi)}$. By equation 17a), the bending prestress is identical for all three mechanical models, and is written as $\bar{M}_{1}^{0}=1-\kappa_{\mathrm{n}} r_{0}=1-\frac{1}{1-\xi}$.

The values of the dimensionless moduli $t$ and $g$ are given in table 2 In particular, for the ribbon, $t=t_{\text {rib }}$ is now a function of the unfolding parameter: $t_{\text {rib }}=2\left(1-\nu \kappa_{\mathrm{n}} r_{0}\right)=2\left(1-\frac{\nu}{1-\xi}\right)$. This modulus reaches zero when $\xi=1-\nu$, corresponding to a curvature $\omega_{1}^{0}=\nu \kappa_{\mathrm{n}}$. In view of this, we expect that the ribbon model predicts an instability before $\xi$ reaches the value $(1-\nu)$. For materials having a positive Poisson's ratio $\nu$ where $1-\nu<1$, the instability is expected to occur well before the ribbon completely flattens. 


\subsection{Linear stability analysis of the unfolding semicircle}

A linear stability analysis of the circular configuration is now carried out by specifying the linearized equations of motion derived in $\$ 4$ for the particular geometry of figure 5 and for the static case, $\bar{\Omega}=0$. This yields the linearized equations of equilibrium in the interior, which are linear ordinary differential equations in the twelve amplitudes $\left(\hat{x}_{i}(\bar{S}), \hat{\psi}_{i}(\bar{S}), \hat{R}_{i}(\bar{S}), \hat{M}_{i}(\bar{S})\right)$ with $i=1,2,3$. These equations are supplemented by the relevant boundary conditions, namely $\left(\hat{x}_{1}, \hat{R}_{2}, \hat{R}_{3}, \hat{\psi}_{1}, \hat{\psi}_{2}, \hat{\psi}_{3}\right)_{S=0}=\left(\hat{x}_{1}, \hat{R}_{2}, \hat{R}_{3}, \hat{\psi}_{1}, \hat{\psi}_{2}, \hat{\psi}_{3}\right)_{\bar{S}=\bar{L}}=\mathbf{0}$. Here, the indices refer to the labelling of the material frame of the base circular solution, see figure 5 the indices 2 and 3 refer to the plane $(O x y)$, while the index 1 refers to the transverse direction $(\mathrm{Oz})$.

The base solution is invariant by mirror symmetry with respect to the $(O x y)$ plane. As a result, the equations for the linear stability decouple into a set of equations for the quantities $\left(\hat{x}_{2}, \hat{x}_{3}, \hat{\psi}_{1}, \hat{R}_{2}, \hat{R}_{3}, \hat{M}_{1}\right)$ on one hand, and another set for the quantities $\left(\hat{x}_{1}, \hat{\psi}_{2}, \hat{\psi}_{3}, \hat{R}_{1}, \hat{M}_{2}, \hat{M}_{3}\right)$ on the other hand; see $\S 5.8$ of reference 2 for details. These two sets of equations characterize in-plane modes of instability involving bending, and out-of-plane modes involving twisting, respectively — the so-called 'ridge mode' and 'centerline mode' in reference [2], respectively. This decoupling is recovered in the analysis of $m$-covered circular solutions later on in 8 .

A detailed analysis shows that the unfolding ribbon first becomes unstable by twisting out of plane. Therefore, to simplify the presentation, we assume that the quantities, $\left(\hat{x}_{2}, \hat{x}_{3}, \hat{\psi}_{1}, \hat{R}_{2}, \hat{R}_{3}, \hat{M}_{1}\right)$ are all identically zero. We cancel the corresponding terms in the decomposition given in equation (18), and insert the result into the linearized equations of motion 16 , making use of the differentiation rule in equation $(19)$. Setting $\bar{\Omega}=0$, we find that

$$
\begin{aligned}
\hat{x}_{1}^{\prime}-\hat{\psi}_{2} & =0, \\
g \hat{\psi}_{2}^{\prime}+\left(\bar{M}_{1}^{0}-g\right) \hat{\psi}_{3}-\hat{M}_{2} & =0, \\
t \hat{\psi}_{3}^{\prime}-\left(\bar{M}_{1}^{0}-t\right) \hat{\psi}_{2}-\hat{M}_{3} & =0, \\
\hat{M}_{2}^{\prime}-\hat{M}_{3}+\hat{R}_{1} & =0, \\
\hat{M}_{3}^{\prime}+\hat{M}_{2} & =0, \\
\hat{R}_{1}^{\prime} & =0,
\end{aligned}
$$

where $\hat{x}_{1}(\bar{S}), \hat{\psi}_{2}(\bar{S}), \hat{\psi}_{3}(\bar{S}), \hat{M}_{2}(\bar{S}), \hat{M}_{3}(\bar{S}), \hat{R}_{1}(\bar{S})$ are functions of the dimensionless arclength $\bar{S}=\frac{S}{L}$. The boundary conditions are

$$
\left(\hat{x}_{1}(0), \hat{\psi}_{2}(0), \hat{\psi}_{3}(0), \hat{x}_{1}(\bar{L}), \hat{\psi}_{2}(\bar{L}), \hat{\psi}_{3}(\bar{L})\right)=\mathbf{0},
$$

where the rescaled arclength is $\bar{L}=L \omega_{1}^{0}=\theta_{\mathrm{n}}(1-\xi)$.

When the expressions of $\bar{M}_{1}^{0}, \bar{L}, g$ and $t$ in terms of $\xi$ and $\nu$ are inserted (here, we use the expressions of $g$ and $t$ relevant to any of the three mechanical models under consideration), equation (20) becomes a linear eigenvalue problem for $\xi$ 
depending on the parameters $\left(\theta_{\mathrm{n}}, \nu\right)$. For a non-trivial solution, these eigenvalues are marginally stable modes, and indicate a bifurcation from the unbuckled (planar, circular) solution branch. An closed-form solution is available because the coefficients of the equations are constant, but that solution turns out to be lengthy and tedious to present. Instead, we solve this problem numerically using a linear shooting method. We call $\mathbf{S}\left(\theta_{\mathrm{n}}, \nu, \xi\right)$ the determinant of the shooting matrix; according to the shooting method [17, the entries in the shooting matrix are found by integrating an initial value problem for the differential system in equation 20. The cancellation of the determinant $\operatorname{det} \mathbf{S}\left(\theta_{\mathrm{n}}, \nu, \xi\right)$ indicates the existence of a marginally stable mode.

The numerical results for the linear stability of an unfolding semicircle are shown in figure 6. For the ribbon model, an instability occurs slightly before the tangent twisting modulus cancels, $t_{\text {rib }}=0$, i.e. slightly before $\xi$ reaches the value $\xi=1-\nu$, as anticipated earlier. The critical value of $\xi$ is numerically very close to $1-\nu$ : the difference is as small as $\Delta \xi=.004$ for $\nu=.3$, as shown in figure 6 a' (even though this difference is a function of Poisson's ratio and of the initial angular span, $\theta_{\mathrm{n}}$, it always stays of this order).

By contrast, the two rod models predict an instability at a much higher value of the unfolding parameter, as indicated in figure 6 b,c. The instability typically does not take place before $\xi$ reaches $\approx 2$, corresponding to a circle that has been fully everted, i.e. has been flattened out and then bent the other way around until it has become mirror-symmetric to the original one.

In summary, the out-of-plane instabilities are predicted at very different unfolding angles by the ribbon model on one hand, and by the rod models on the other hand. This is evident from the very different positions of the stability boundaries in the panel ' $a$ ' of figure 6, as compared to those in panels ' $b$ ' and ' $c$ '. The early instability of the ribbon model is triggered by the change of sign of the tangent twisting modulus - no such change of sign takes place for the rod models.

\subsection{Simulations by the finite element method}

In order to test our theoretical predictions, we performed computational simulations using a finite element analysis for thin elastic shells using the commercially available software package ABAQUS [8]. The semi-circle $\left(\theta_{\mathrm{n}}=\pi\right)$ is formed as a thin strip using four-noded S4R5 shell elements, with 20 elements across the width and 200 around its length. The previous boundary conditions are captured exactly: the ends are free to rotate about an axis parallel to the width direction, and no other rotations are permitted; one end is restrained against linear translation and the other end can only displace in a direction between the ends. The strip is loaded by imposing end-wise, in-plane rotations, and uniform flattening without end-wise reaction forces is accomplished by the ends freely moving relative to one another (they move apart for $\theta>\pi / 2$, and then together when $\theta$ is larger). The shell elements can bend and stretch; we use a thickness parameter than is only a small fraction of the width, see figure 7 , implying that the equilibrium configurations are close to inextensible. The high density 

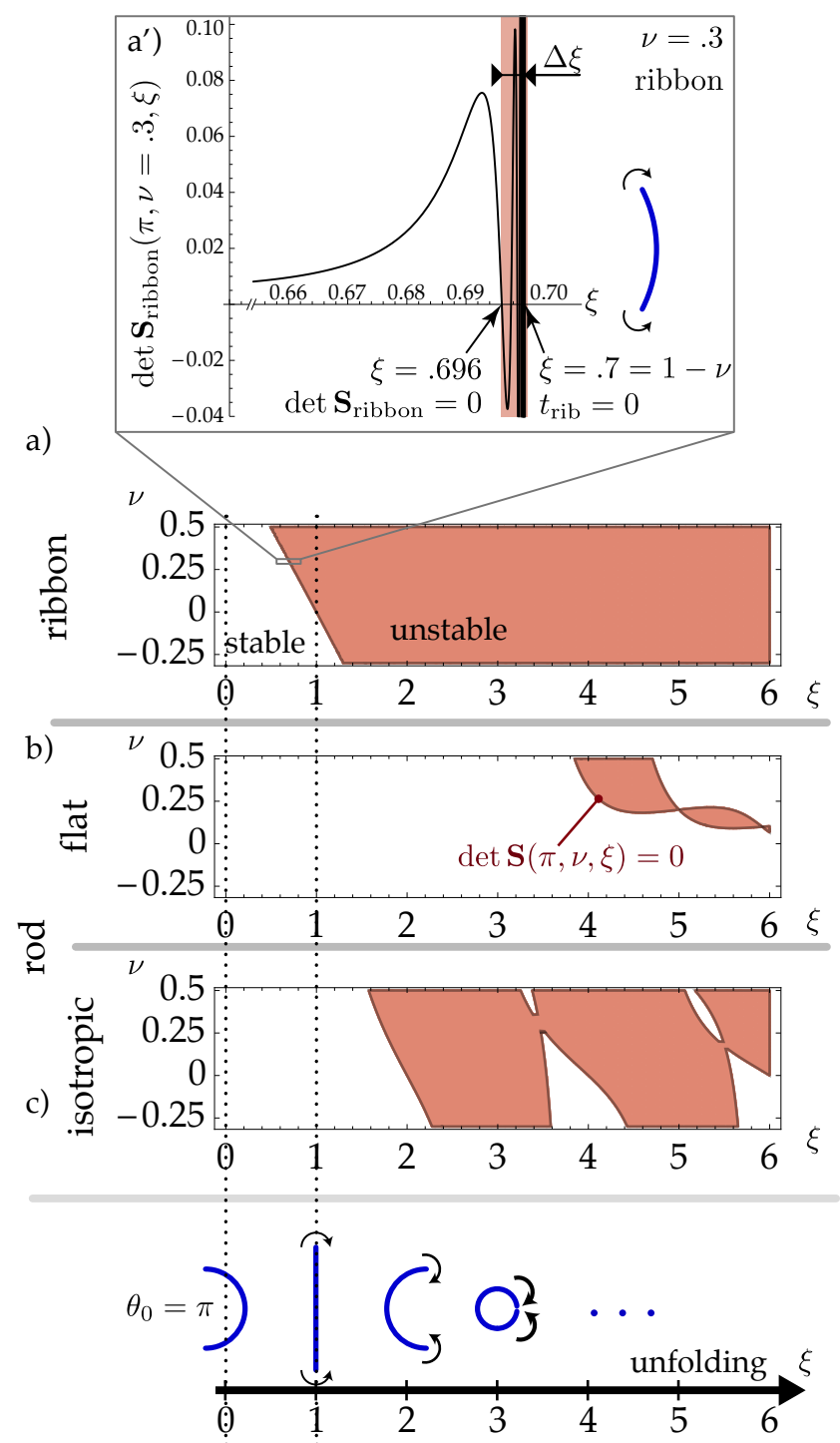

Figure 6: Linear stability analysis of the unfolding semicircle $\left(\theta_{\mathrm{n}}=\pi\right)$, as predicted (a) by the ribbon model, (b) by the flat rod model, and (c) by the isotropic rod model. The ribbon model predicts a much lower instability threshold than the two other models. The red domains correspond to a change of sign of the determinant of the shooting matrix $\mathbf{S}\left(\theta_{\mathrm{n}}, \nu, \xi\right)$ : along its boundary, a marginally stable twisting mode exists. (a') For the ribbon model, the first instability, corresponding to the first root of the determinant of $S$, occurs slightly below the value $\xi=1-\nu$ where the tangent twisting modulus cancels: the gap between the two is as small as $\Delta \xi=.004$ for $\nu=.3$. 


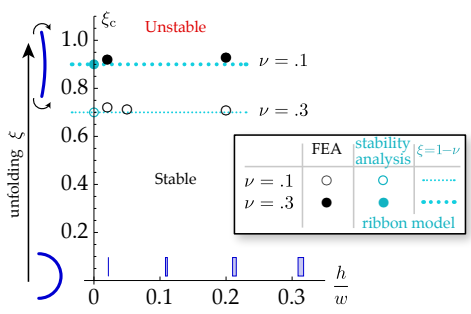

Figure 7: Stability analysis of the unfolding semi-circular ribbon $\left(\theta_{\mathrm{n}}=\pi\right)$. Comparison of the finite element simulation (black symbols) to the ribbon model (light blue): full stability analysis (symbols), and approximate threshold $\xi=$ $1-\nu$ where the twisting modulus cancels, $t_{\text {rib }}=0$, (dotted lines).

of elements across the width of strip ensures that all stresses are accurately resolved.

The material is chosen to be linear elastic, with the Young's modulus of steel, $210 \mathrm{GPa}$, and three width-to-thickness ratios, 5, 20 and 50, are considered, along with two values, $\nu=.1$ and $\nu=.3$, of Poisson's ratio. The initial radius is $100 \mathrm{~mm}$ and the width is always $w=5 \mathrm{~mm}$, giving a length-to-width ratio $L / w=62.8$. The analysis follows two steps. First, the strip is unbent to a given end rotation, which is then held fixed with all other nodal displacements being free. Second, a linear perturbation analysis is performed where the value of the primary (lowest) eigenvalue is extracted. These steps are repeated for a range of end rotations where the aim is to capture the eigenvalue performance of the strip as a function of end rotation, width and Poisson's ratio: the value at which an eigenmode first becomes unstable is detected, and this critical value is plotted as a function of the aspect-ratio $h / w$ and of Poisson's ratio $\nu$. The results have been collated in figure 7. A good agreement is obtained with the linear stability analysis of the one-dimensional ribbon model. These finite element simulations confirm the existence of buckling instabilities of undercurved strips, triggered by the change of sign of their tangent twisting modulus.

\section{Stability of overcurved and undercurved $m$ - covered strips}

\subsection{Geometry}

We return to the experiments of $\$ 2$ concerning the stability of $m$-covered loops. The geometry is sketched in figure 8 . The natural curvature is denoted by $\kappa_{\mathrm{n}}$. The strip is closed and the single loop $(m=1)$ is used as the reference configuration. It is then bent into the $m$-covered strip sketched in figure $8 \mathrm{~b}$, using the folding sequence of figure 2. Topology shows that the single loop can be deformed by means of continuous deformations into an $m$-covered configuration 
(a)

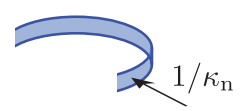

(b)

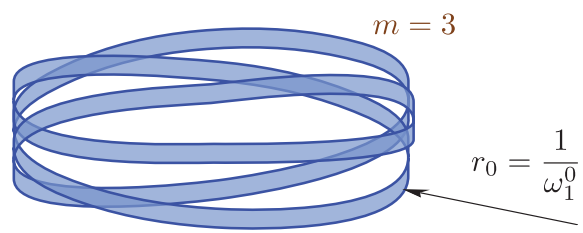

Figure 8: A multiloop. (a) A small chunk of the loop shown in natural configuration, revealing the natural curvature $\kappa_{\mathrm{n}}$. (b) An $m$-covered strip with covering index $m=3$, exhibiting over-curvature $\left(\omega_{1}^{0}>\kappa_{\mathrm{n}}\right)$ or under-curvature $\left(\omega_{1}^{0}<\kappa_{\mathrm{n}}\right)$. The loop has been disentangled to help visualization but in our analysis selfpenetration is allowed and a perfectly planar, self-intersecting configuration is considered.

only if $m$ is an odd integer - see figures 1 and 2 We therefore restrict our attention to odd integers $m$; in addition, the $m$-covered configuration involves self-penetration if $m>1$ but we ignore self-penetration entirely in our analysis.

We now analyze the stability of the $m$-covered strip, first by means of a linear stability analysis $(\$ 6.2)$, and then by calculating the post-bifurcation solutions numerically $(\$ 6.3)$. Let $L$ be the length of the strip. The radius of the $m$ covered configuration is $r_{0}=\frac{L}{2 \pi m}$, which is said to be undercurved if $\omega_{1}^{0}<\kappa_{\mathrm{n}}$, i.e. $m<\frac{\kappa_{\mathrm{n}} L}{2 \pi}$, and overcurved if $\omega_{1}^{0}>\kappa_{\mathrm{n}}$, i.e. $m>\frac{\kappa_{\mathrm{n}} L}{2 \pi}$.

\subsection{Linear stability analysis}

The planar, $m$-covered configuration is a particular case of a circular solution: we use it as the base state of the general stability analysis carried out in 4

Owing to the rotational invariance of the base state, we consider a vibration mode with dimensionless wavenumber $\bar{k}$, corresponding to a physical wavenumber $k=\omega_{1}^{0} \bar{k}$. We denote by $\Re$ the real part of a complex number: the components of the perturbations in the directors basis are sought as

$$
\left(\begin{array}{c}
\hat{x}_{j}(\bar{S}) \\
\hat{\psi}_{j}(\bar{S}) \\
\hat{R}_{j}(\bar{S}) \\
\hat{M}_{j}(\bar{S})
\end{array}\right)=\Re\left[e^{i \bar{k} \bar{S}}\left(\begin{array}{c}
x_{j}^{\dagger} \\
\psi_{j}^{\dagger} \\
R_{j}^{\dagger} \\
M_{j}^{\dagger}
\end{array}\right)\right]
$$

in terms of complex amplitudes

$$
\hat{\mathbf{y}}=\left(\hat{x}_{1}, \hat{x}_{2}, \hat{x}_{3}, \hat{\psi}_{1}, \hat{\psi}_{2}, \hat{\psi}_{3}, \hat{R}_{1}, \hat{R}_{2}, \hat{R}_{3}, \hat{M}_{1}, \hat{M}_{2}, \hat{M}_{3}\right) .
$$

Since the ribbon is closed, the perturbations are periodic after $m$ turns. This imposes a quantification condition for $\bar{k}$ : there exists some integer $n$ such that

$$
\bar{k}=\frac{n}{m} \text {. }
$$


The difference in phase after $m$ turns, $\bar{k} \bar{L}=\frac{n}{m} \frac{L}{r_{0}}=2 \pi n$ is then a multiple of $2 \pi$.

We insert the harmonic form of the perturbations in equation (21) into equation (18), and then into the linearized equations of motion (16), making use of the differentiation rule in equation (19). The resulting linearized equations of motion take the form

$$
\mathbf{A}\left(\bar{M}_{0}^{1}, g, t ; \bar{k}, \bar{\Omega}^{2}\right) \cdot \hat{\mathbf{y}}=\mathbf{0},
$$

where

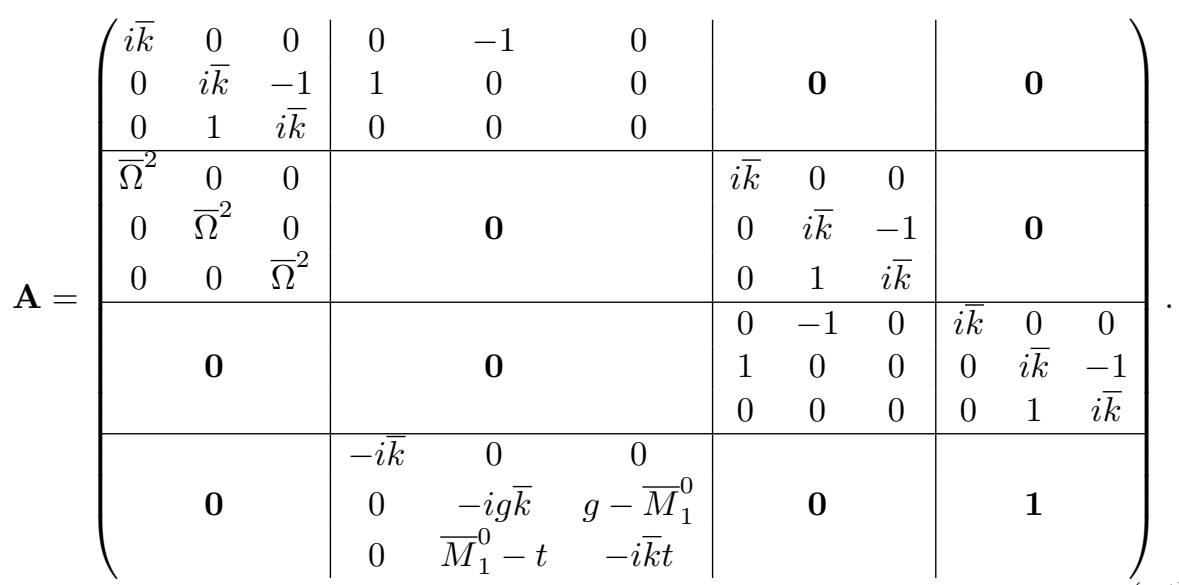

Note that for $\bar{\Omega}=0$ it is possible to write $\mathbf{A}$ as a diagonal block matrix by reordering the rows and columns - this is the decoupling discussed earlier in $\$ 5.3$, arising from the symmetry of the base state.

The dispersion relation reads $\operatorname{det} \mathbf{A}\left(\bar{M}_{0}^{1}, g, t ; \bar{k}, \bar{\Omega}^{2}\right)=0$. Factoring the determinant, we obtain

$$
\operatorname{det} \mathbf{A}=g\left(\left(1+\bar{k}^{2}\right) \bar{\Omega}^{2}-\bar{k}^{2}\left(1-\bar{k}^{2}\right)^{2}\right)\left(c \bar{\Omega}^{2}-b \bar{k}^{2}\left(1-\bar{k}^{2}\right)\right),
$$

where

$$
\begin{aligned}
b & =\frac{\left(\bar{M}_{1}^{0}\right)^{2}}{g}-\left(1+\frac{t}{g}\right) \bar{M}_{1}^{0}+t\left(1-\bar{k}^{2}\right), \\
c & =1+\frac{\bar{k}^{2} t-\bar{M}_{1}^{0}}{g} .
\end{aligned}
$$

The roots $\bar{\Omega}^{2}$ of the dispersion relation $\operatorname{det} \mathbf{A}=0$ are the numbers $\bar{\Omega}^{2}=$ $\left(\bar{\Omega}^{2}\right)_{1}$ and $\bar{\Omega}^{2}=\left(\bar{\Omega}^{2}\right)_{2}$ defined by

$$
\begin{aligned}
& \left(\bar{\Omega}^{2}\right)_{1}=\frac{\bar{k}^{2}\left(1-\bar{k}^{2}\right)^{2}}{1+\bar{k}^{2}}, \\
& \left(\bar{\Omega}^{2}\right)_{2}=\frac{b \bar{k}^{2}\left(1-\bar{k}^{2}\right)}{c} .
\end{aligned}
$$




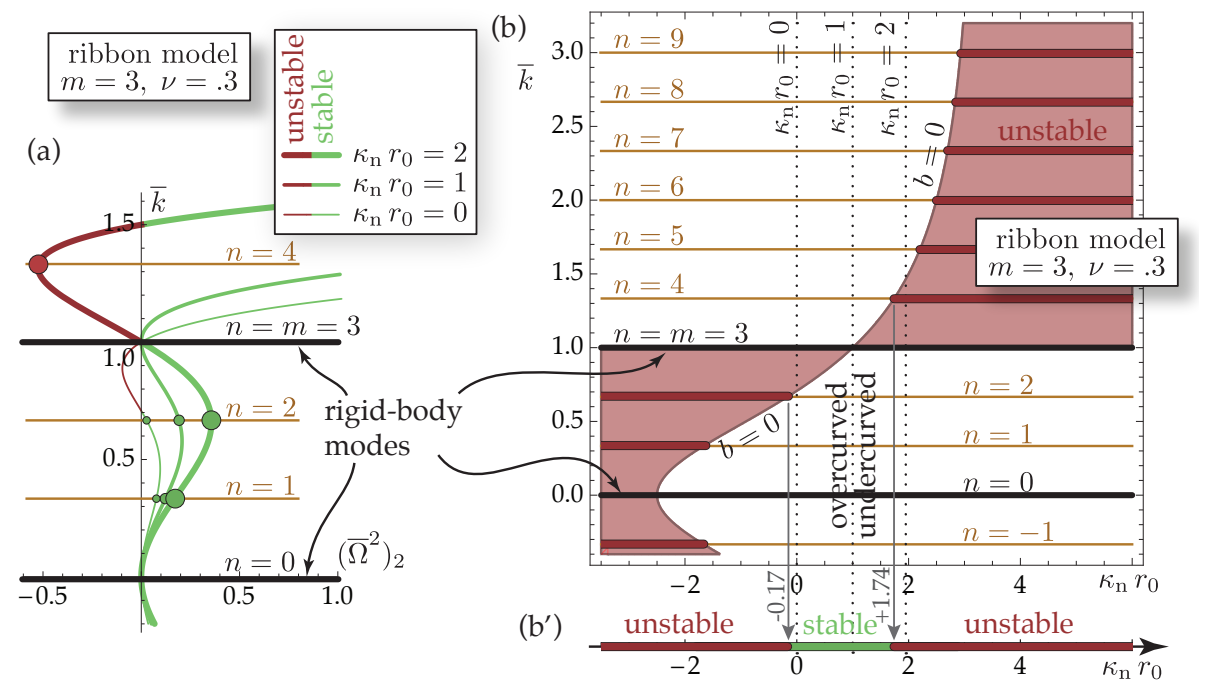

Figure 9: Linear stability analysis of $m$-covered configurations with $m=3$ and $\nu=.3$, based on the ribbon model. (a) Dependence of the most negative squared angular velocity $\left(\bar{\Omega}^{2}\right)_{2}$ on the wavenumber. (b) Graphical determination of the stability of $m$-covered ribbons for fixed $\nu=.3$ as a function of the parameter $\kappa_{\mathrm{n}} r_{0}$ using the ribbon model. Unstable modes corresponds to the thick, horizontal, dark red segments. (b') Configurations are stable when no unstable mode exists (light green segment), and unstable otherwise (dark red segments). See the text for details.

The first root $\left(\bar{\Omega}^{2}\right)_{1}$ being always positive, the stability of the ribbon is governed by the sign of the second root. This sign changes when $\bar{k}=0$, when $\bar{k}= \pm 1$, when $b=0$, or when $c=0$ : these four equations yield the four possible types of boundaries surrounding the regions of stability in figures $9 \mathrm{~b}$ and 10 .

The quantity $\left(\bar{\Omega}^{2}\right)_{2}$ is a function of $\bar{k}, b$ and $c$. On using equations $(25), b$ and $c$ can be expressed in terms of $\bar{M}_{1}^{0}, g$ and $t$. Using table 2 and equation (17a), we can finally express $\left(\bar{\Omega}^{2}\right)_{2}$ as a function of $\bar{k}, \nu$ and $\left(\kappa_{\mathrm{n}} r_{0}\right)$; there are three such expressions, one for each mechanical model.

In figure 9 a, we plot $\left(\bar{\Omega}^{2}\right)_{2}$ predicted by the ribbon model for $\nu=.3$ and for $\kappa_{\mathrm{n}} r_{0}=0$ (naturally straight ribbon, overcurved configuration, $\bar{M}_{1}^{0}>0$ ), $\kappa_{\mathrm{n}} r_{0}=$ 1 (natural configuration, $\bar{M}_{1}^{0}=0$ ), and $\kappa_{\mathrm{n}} r_{0}=2$ (undercurved configuration, $\left.\bar{M}_{1}^{0}<0\right)$. Stable modes corresponding to $\left(\bar{\Omega}^{2}\right)_{2}>0$ are shown in light green, and unstable modes corresponding $\left(\bar{\Omega}^{2}\right)_{2}<0$ are shown in dark red. For a given value of the covering index $m$ (in this case, $m=3$ ) the values of $\bar{k}$ satisfying the quantification condition $(22)$ are shown as horizontal lines. The modes corresponding to $\bar{k}=0$ and $\bar{k}= \pm 1$ (i.e. $n=0$ and $n= \pm m$ ) make both $\left(\bar{\Omega}^{2}\right)_{1}$ and $\left(\bar{\Omega}^{2}\right)_{2}$ vanish: these are rigid-body modes, which we represent by thick black 
lines. All other modes are non-rigid, and are shown using a thin brown line. Vibration modes are denoted by small disks: stable modes correspond to the intersection of a horizontal brown line with the green portion of a curve, and unstable vibration modes to an intersection with the red portion of a curve.

The influence of the parameter $\kappa_{\mathrm{n}} r_{0}$ on the stability is grasped by the alternative presentation of the same data proposed in figure $9 \mathrm{~b}$. For a fixed value of $\nu$ and a specific mechanical model (in this case, $\nu=.3$ with the ribbon model), the region where $\left(\bar{\Omega}^{2}\right)_{2}<0$ is shaded in the plane $\left(\kappa_{\mathrm{n}} r_{0}, \bar{k}\right)$. The representative values $\kappa_{\mathrm{n}} r_{0}=0,1,2$ used earlier now correspond to the vertical dotted lines. Unstable modes lie at the intersection of horizontal brown lines representing the quantization condition for $\bar{k}$ with the shaded regions where $\left(\bar{\Omega}^{2}\right)_{2}<0$. The $m$-covered ribbon is stable for a given value of $\kappa_{\mathrm{n}} r_{0}$ if, and only if, no unstable mode exists: this test is carried out graphically by projecting vertically the red segments onto the $\left(\kappa_{\mathrm{n}} r_{0}\right)$-axis in figure $9 \mathrm{~b}$ '. For this particular set of parameters, the $m$-covered ribbon appears to be stable in a single interval, $-.17<\kappa_{\mathrm{n}} r_{0}<+1.74(m=3, \nu=.3$, ribbon model $)$. This solves the linear stability problem of the $m$-covered ribbon, with $\nu=.3$ and $m=3$. The same analysis is repeated in figure 10 for different values of $m$, and for the rod models as well. Note that we recover the results of [15] when we use the rod models, and the results of [6] in the particular case of a rod making a single loop, $m=1$; the results concerning the stability of the ribbon model are novel.

We make a few comments on these results. First, we check our analysis by confirming that the natural configuration is always stable: the stability regions in figure $10 \mathrm{a}$ '- $\mathrm{c}$ ' all contain the point $\kappa_{\mathrm{n}} r_{0}=1$, corresponding to a zero prestress $\bar{M}_{1}^{0}=0$.

Secondly, we observe that the ribbon model is, generally speaking, less stable than the flat rod model. This difference is particularly marked for the simple loop configuration $(m=1)$, which is stable for $\kappa_{\mathrm{n}} r_{0}<2.50$ with the ribbon model, and up to $\kappa_{\mathrm{n}} r_{0}<5.62$ with the flat rod model. This large difference in the upper bounds for $\kappa_{\mathrm{n}} r_{0}$ can be traced back to the presence of a vertical asymptote to the curve, $b=0$, bounding the stability region for the ribbon model (shown as the vertical thick dashed line in figure 10a); by contrast, no such asymptote exists for the flat rod model and the upper part of the curve, $b=0$, is much flatter in figure $10 \mathrm{~b}$ than in 10 . Starting from equation $(25 \mathrm{~b})$, it is easy to show that the vertical asymptote is where the tangent twisting modulus cancels, $t_{\mathrm{rib}}=0$, and corresponds to $\kappa_{\mathrm{n}} r_{0}=1 / \nu$. Therefore, the change of the sign of the tangent twisting modulus $t_{\text {rib }}$ is responsible for the less stable behavior of the ribbon model as compared to the rod models - a similar conclusion as for the earlier unfolding semicircle in \$5.1.

Thirdly, we observe that the isotropic rod model is less stable than the flat rod model. Recall that the difference between these models is that the flat rod is subjected to the kinematical constraint $\omega_{2}=0$, as captured by the statement $g_{\text {flat }}=0$. Unsurprisingly, the flat rod model has more flexibility to explore unstable modes, is also more stable. As a particular case, we recover known results [15] concerning the stability of naturally straight isotropic rods, see the 

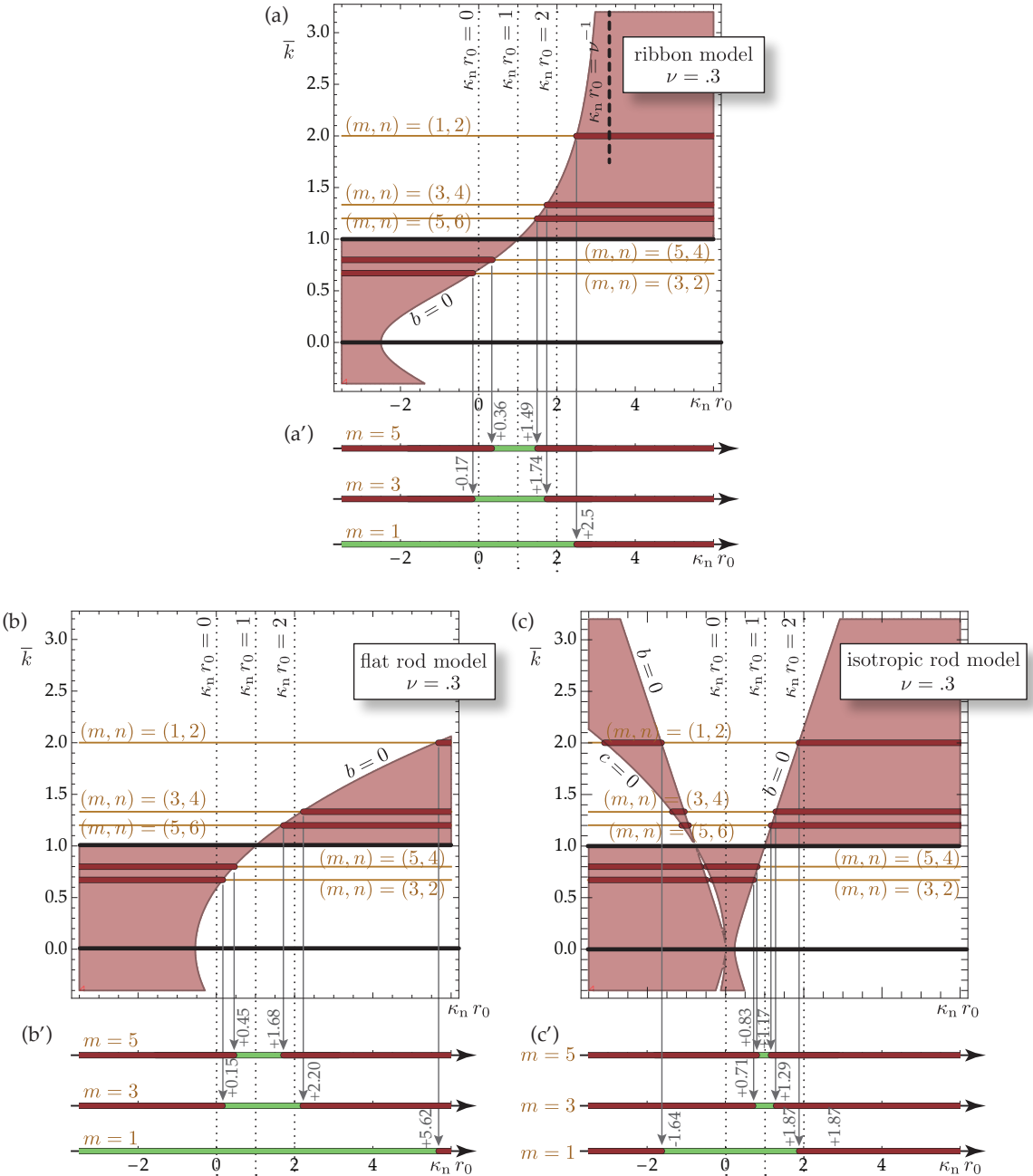

Figure 10: Linear stability analysis of multiloop configurations, as predicted by the different mechanical models, for $\nu=.3$ and for various covering indices $m$. We recover the results of [15] for the rod models as particular cases. 
dotted line $\kappa_{\mathrm{n}} r_{0}=0$ in figure $10 \mathrm{~b}-\mathrm{b}$ ': for $\nu=.3, m$-covered configurations of a naturally straight isotropic rod are found to be unstable for all values of $m$, except for $m=1$.

Finally, we found more boundaries to the stability domains in figure 10 e for the isotropic rod model, which has a finite second bending modulus $g_{\text {iso }}<\infty$, than in figure 10 $\mathrm{a}-\mathrm{b}$ for the flat rod and ribbon models for which $g_{\text {flat }}=g_{\text {rib }}=$ $\infty$. This can be explained as follows. The equations $b=0$ and $c=0$ defining the boundaries are polynomial equations for $\kappa_{\mathrm{n}} r_{0}$ and $\bar{k}$ when the expressions of $\bar{M}_{1}^{0}=1-\kappa_{\mathrm{n}} r_{0}$ and of $t$ and $g$ from table 2 are inserted into equations $25 \mathrm{~b}$ 25c. Their order is decreased when $g$ is infinite. The equation $b=0$ is a quadratic equation for $\kappa_{\mathrm{n}} r_{0}$ when $g$ is finite but a linear equation for $g=\infty$, and the equation $c=0$ is a linear equation for $\kappa_{\mathrm{n}} r_{0}$ if $g$ is finite and has no root if $g$ is infinite (it does not depend on $\kappa_{\mathrm{n}} r_{0}$ then). Correspondingly, figures $10 \mathrm{a}-\mathrm{b}$ (ribbon and flat rod models) have a single $b=0$ branch and no $c=0$ branch, and figure 10 has two $b=0$ branches and another $c=0$ branch (as in reference [15]).

Along the boundaries $b=0$ and $c=0$ marginally stable modes exist, which correspond to a loss of stability of the planar, $m$-covered configuration. There, the determinant $\operatorname{det} \mathbf{A}\left(\bar{M}_{0}^{1}, g, t ; \bar{k}, \bar{\Omega}^{2}=0\right)$ nullifies and the marginally stable modes can be found by calculating the null vectors $\hat{\mathbf{y}}$ of $\mathbf{A}$. As noted earlier, these modes are rigid-body modes for $\bar{k}=0$ (translation along the axis $\mathbf{e}_{z}$, and rigid rotation about the axis $\mathbf{e}_{z}$ ) and $\bar{k}= \pm 1$ (translations along the directions $\mathbf{e}_{x}$ and $\mathbf{e}_{y}$, and rigid rotations about these directions); for $b=0$, the marginally stable modes are out-of-plane modes similar to that studied earlier in 5.3 . finally, the boundary $c=0$, which is only encountered with the isotropic rod model $\left(g_{\text {iso }}<\infty\right)$, corresponds to a marginal mode involving locally a pure-twist deformation about the tangent 5 .

Returning to the ribbon model, we note that an analytical expression of the stability boundaries can be obtained. Rewriting the equation $b=0$ in terms of the parameter $\kappa_{\mathrm{n}} r_{0}$ and using the quantification condition 22 for $\bar{k}$, we express the roots of $b=0$ as $\kappa_{\mathrm{n}} r_{0}=K_{\mathrm{rib}}^{m, n}$, where

$$
K_{\mathrm{rib}}^{m, n}=\frac{1}{\nu}\left(1-\frac{1-\nu}{2 \nu\left(\left(\frac{n}{m}\right)^{2}-1\right)+1}\right) \quad \text { (ribbon model). }
$$

All stability boundaries shown in figure 10 ' ' correspond to particular values of $K_{\mathrm{rib}}^{m, n}$ listed table 3 in fact, the values $K_{\mathrm{rib}}^{m, m \pm 1}$ shown in boldface in the table are the critical values of the parameter $\kappa_{\mathrm{n}} r_{0}$ found earlier in figure 10a'. We conclude that, according to the ribbon model, the $m$-covered ribbon is linearly

\footnotetext{
${ }^{4}$ We just found that the stability boundaries for the ribbon model are either rigid-body modes, or out-of-plane modes $(b=0)$ but never in-plane modes $(c=0$ has no root for $g=\infty)$. This confirms the assumption made earlier in 5.3 that the stability of the ribbon model can be analyzed assuming that the perturbations associated with in-plane modes are zero.

${ }^{5}$ We have ignored the rotational inertia in equation $16 \mathrm{c}$ for the balance of angular momentum and, as a result, pure twist modes are associated with zero inertia and an infinite frequency: this is why $c=0$ makes $\left(\bar{\Omega}^{2}\right)_{2}$ infinite in equation $26 \mathrm{a}$.
} 
Table 3: Upper and lower stability bounds for $m$-covered configurations based on the linear stability analysis of the ribbon model for $\nu=.3$, as predicted by equation (27). Values of $K_{\mathrm{rib}}^{m, n}={ }^{\prime} 1$ '' in italics correspond to rigid-body modes $(n=m)$ and should be ignored. Values in boldface $(n=m \pm 1)$ correspond to critical values of $\kappa_{\mathrm{n}} r_{0}$ where stability is lost. Other values correspond to higherorder modes appearing when the base circular solution is already unstable.

\begin{tabular}{c|ccccccc}
$K_{\text {rib }}^{m, n}$ & $n=1$ & $n=2$ & $n=3$ & $n=4$ & $n=5$ & $n=6$ & $n=7$ \\
\hline$m=1$ & 1. & $\mathbf{2 . 5 0}$ & 2.93 & 3.10 & 3.18 & 3.23 & 3.26 \\
$m=3$ & -1.67 & $\mathbf{- 0 . 1 7}$ & 1. & $\mathbf{1 . 7 4}$ & 2.20 & 2.50 & 2.70 \\
$m=5$ & -2.17 & -1.37 & -0.45 & $\mathbf{0 . 3 6}$ & 1. & $\mathbf{1 . 4 9}$ & 1.85 \\
$m=7$ & -2.33 & -1.86 & -1.24 & -0.58 & 0.03 & $\mathbf{0 . 5 6}$ & 1.
\end{tabular}

stable for $\kappa_{\mathrm{n}} r_{0}<K_{\mathrm{rib}}^{1,1}=2.5$ for $m=1$, and for $K_{\mathrm{rib}}^{m, m-1}<\kappa_{\mathrm{n}} r_{0}<K_{\mathrm{rib}}^{m, m+1}$ for $m \geq 3$. Similar expressions for the stability boundaries of the rod models have been obtained in reference 15 .

In summary, we have analyzed the linear stability of $m$-covered configurations of an elastic strip, ignoring self-penetration. The ribbon model yields results that are qualitatively similar to those of the flat rod model. Quantitatively, however, the buckling threshold in the undercurved case (i.e. for large positive values of $\kappa_{\mathrm{n}} r_{0}$ ) are substantially lower with the ribbon model than with the flat rod model, especially for $m=1$ : this difference can be attributed mainly to the change of sign of the tangent twisting modulus, $t_{\mathrm{rib}}$, at $\kappa_{\mathrm{n}} r_{0}=1 / \nu$ (thick dashed line in figure 10). All instabilities of the ribbon involve an out-of-plane mode.

\subsection{Numerical post-bifurcated solutions}

To validate and complement this stability analysis, we solved numerically the post-bifurcation equilibrium solutions for a closed elastic ribbon. To do this, we solved equations (1) 4) together with the constitutive law for an elastic ribbon in equation (9) and with periodic boundary conditions. We used AUTO-07p [4, a library which implements the arc-length continuation method [14]. We started from an $m$-covered planar configuration, detected bifurcations along this fundamental branch, and followed the various bifurcated solution branches emanating from it. The branches are plotted in a plane whose coordinates are the dimensionless natural curvature $\frac{\kappa_{\mathrm{n}} L}{2 \pi}$ and the conjugate quantity $\kappa^{*}=-\frac{\partial \mathcal{E}}{\partial \kappa_{\mathrm{n}}}$ : our diagram is distinguished in the sense of [13. In dimensionless form, and after using the definition of the ribbon energy in equation $(7), \bar{\kappa}^{*}$ is given by

$$
\bar{\kappa}^{*}=-\frac{1}{2 \pi B} \frac{\partial \mathcal{E}}{\partial \kappa_{\mathrm{n}}}=\frac{1}{2 \pi} \int_{0}^{L} \omega_{1}\left(1+\nu\left(\frac{\omega_{3}}{\omega_{1}}\right)^{2}\right) \mathrm{d} S .
$$

For planar configurations, note that $\omega_{3}=0$ and thus that $\bar{\kappa}^{*}=m$.

A typical branch is represented in figure 11, this is the branch starting off from the simple loop $(m=1)$, and extending the mode of instability $n=2$ 


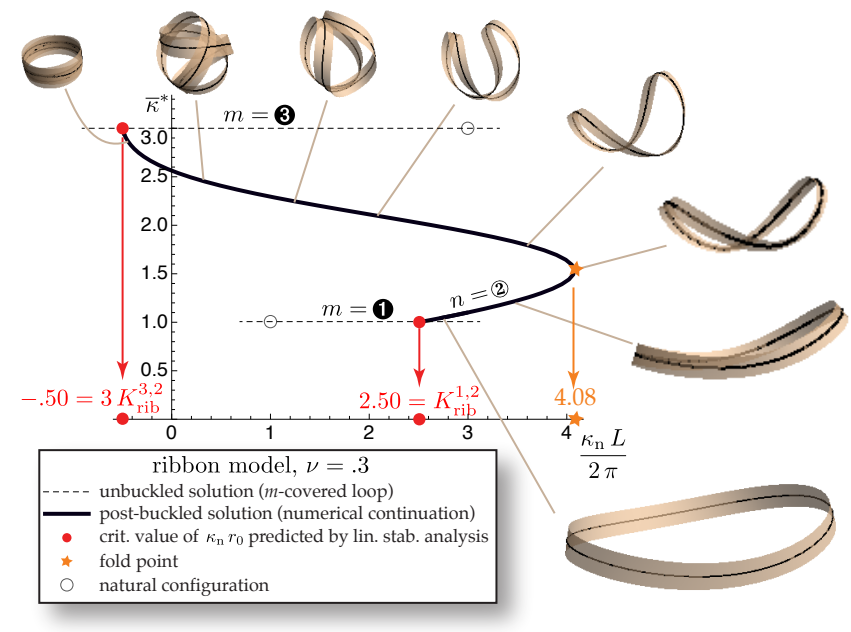

Figure 11: A branch of post-bifurcated equilibrium solutions connecting the simple loop $(m=1)$ to the 3-covered loop $(m=3)$, as simulated by numerical continuation of the ribbon model with $\nu=.3$. The continuation parameter is the dimensionless natural curvature $\kappa_{\mathrm{n}}$ (horizontal axis); the quantity on the vertical axis is the conjugate quantity $\bar{\kappa}^{*}$.

occurring for $\kappa_{\mathrm{n}} r_{0}=K_{\mathrm{rib}}^{1,2}$, i.e. $\kappa_{\mathrm{n}} \frac{L}{2 \pi}=K_{\mathrm{rib}}^{1,2}=2.5$. Above the buckling threshold, the dimensionless natural curvature $\frac{\kappa_{\mathrm{n}} L}{2 \pi}$ first increases, which is typical of a supercritical pitchfork bifurcation. It reaches a maximum value 4.08 at a fold point, and then decreases. Eventually, this branch connects to the triply-covered circular configuration $(m=3)$; the value of the control parameter where this connection occurs is $\frac{\kappa_{\mathrm{n}} L}{2 \pi}=\kappa_{\mathrm{n}}\left(m r_{0}\right)=m K_{\mathrm{rib}}^{m, m-1}=3 K_{\mathrm{rib}}^{3,2}=-.5$. Therefore, the connections of the two endpoints of this branch with the planar, $m$-covered configurations are fully consistent with the linear stability analysis of 6.2 . Also, we note that the sequence of equilibria shown alongside the branch closely resemble the folding sequence shown in figure $2 \mathrm{a}$.

For rods, there exists a similar branch of non-linear solutions $n=2$ connecting the simple loop $(m=1)$ to the triply-covered loop $(m=3)$, but no fold point exists along this branch, see figure 2 in [15]: it is not surprising that the ribbon model, for which the constitutive laws are non-linear, gives rise to new bifurcations (here, a new fold point). As a result, the bifurcation $(m, n)=(1,2)$ a supercritical (continuous) pitchfork bifurcation for rods, while it is a subcritical (discontinuous) pitchfork bifurcation for the ribbon model.

Repeating this continuation procedure for all the other branches emanating from the $m$-covered configurations, we constructed the diagram shown in figure 12. The branch studied in figure 11 appears there, along with many other branches. The stability of the non-planar branches has been established as follows: we first identified the bifurcations from the layout of the branches (such 


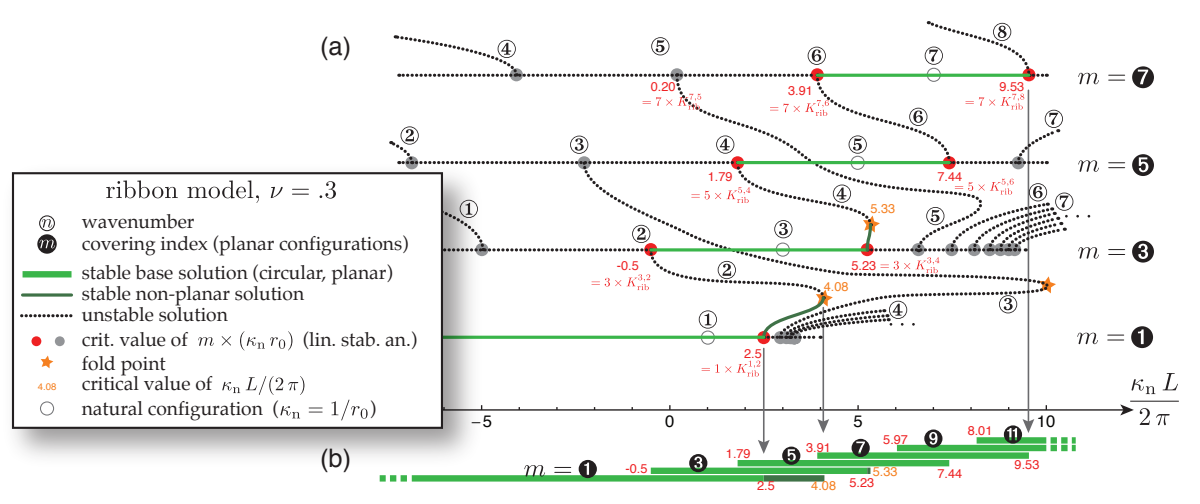

Figure 12: (a) Sketch of the branches of non-linear equilibria of a closed elastic ribbon, for $\nu=.3$. The horizontal axis is the dimensionless natural curvature. The stacked horizontal lines represent the $m$-covered planar configurations, with $m$ increasing from bottom to top. The curves represent the non-planar equilibrium branches connecting planar $m$-covered configurations. These curves were first plotted in the same set of conjugate coordinates as in figure 11, and they disentangled by hand to aid visualization, in such a way that their projection onto the horizontal axis is unaffected; as a result, the vertical axis loosely represents the parameter $\bar{\kappa}^{*}$. Dots denote connections of non-planar branches with planar ( $m$-covered) configurations, as calculated by the linear stability analysis of $₫ 6.2$ The stability of the planar solutions has been concluded based on the analysis of 86.2 The stability on non-planar solutions has been guessed based on the generic scenario of stability exchange at subcritical or subcritical pitchfork bifurcations and at fold points. (b) Domains of existence of stable planar (green) and stable non-planar (dark green) $m$-covered ribbons. 
as a supercritical pitchfork bifurcation for $m=1$ and $\frac{\kappa_{\mathrm{n}} L}{2 \pi}=2.5$, a subcritical pitchfork bifurcation for $m=3$ and $\frac{\kappa_{\mathrm{n}} L}{2 \pi}=-.5$, and fold point along the $n=2$ branch at $\frac{\kappa_{\mathrm{n}} L}{2 \pi}=4.08$ ), and assumed that the generic stability scenario associated with each of these bifurcations is applicable (i.e. the bifurcated branches are stable past a supercritical pitchfork bifurcation, and unstable past a subcritical pitchfork bifurcation; an unstable mode appears across a fold point). The stable branches inferred in this way are summarized in part b of the figure. These stability results would need to be confirmed by a more thorough analysis, such as that done in 13 for the rod model.

A comparison with the bifurcation diagram for the anisotropic rod model, shown in figure 2 of [15], reveals some similarities concerning the general layout of the branches. There are two major differences, however. First, the evolution of the dimensionless curvature $\kappa_{\mathrm{n}}$ along the branches is different: on almost all branches the natural curvature varies monotonously for the anisotropic rod model [15, while along many branches of the ribbon model, the natural curvature first increases, passes through a maximum (fold points, orange stars in the diagram) and then decreases, see figure 12 above. Second, the bifurcations (and the equilibrium branches) for overcurved ribbons cluster near accumulation point, as happens for instance near $\left(\frac{\kappa_{\mathrm{n}} L}{2 \pi}, m\right)=(1,3.33)$ and $\left(\frac{\kappa_{\mathrm{n}} L}{2 \pi}, m\right)=(3,10$. $)$ in figure 12. This is consistent with the fact that the critical value $m K_{\text {rib }}^{m, n}$ of the quantity $\frac{\kappa_{\mathrm{n}} L}{2 \pi}$ converges to $\frac{m}{\nu}$ for $n \rightarrow \infty$ by equation (27). This value is precisely where the tangent twisting modulus cancels; when this happens, all wavenumbers become unstable, hence the accumulation of bifurcations. By contrast, the critical values and the branch of the rod models are evenly spaced [15].

To sum up, we have obtained a complete bifurcation diagram showing the non-linear equilibria of a closed ribbon. As for anisotropic rod models, branches corresponding to non-planar equilibria of a ribbon connect planar solutions of different covering indices $m$ through bifurcations that are well predicted by the linear stability analysis of $\$ 6.2$. However, both the critical values of the natural curvature for the onset of buckling from the planar configuration, and the aspect of the post-buckled branches differ significantly with the ribbon model, as compared to the rod model: the branches of the (more non-linear) ribbon model tend to cluster and they often include fold points where the natural curvature passes through a maximum.

\subsection{Comparison to experiments}

In the experiments of $\$ 2$ the single loop $(m=1)$ has been found to be stable for $\kappa_{\mathrm{n}} r_{0}<3.5$; see table 1 . This value is above the threshold $K_{\text {ribb }}^{1,2}=2.5$ predicted by the linear stability analysis of the ribbon model for $\nu=.3$, which is not surprising in view of the existence of a stable, non-planar branch past this bifurcation (dark green branch bifurcating from $(m, n)=(1,2)$ in figure 12). The experimental threshold $\kappa_{\mathrm{n}} r_{0}=3.5$ is reasonably close to the fold point terminating the stable part of the post-buckled branch at $\kappa_{\mathrm{n}} r_{0}=4.08$. By contrast, the flat rod model predicts a much higher stability limit, namely $\kappa_{\mathrm{n}} r_{0}=5.62$ 
(see figure 10b').

Comparison of the experimental stability limits for $m \geq 3$ is more difficult because of the presence of self-contact and friction, both of which were ignored in the analysis. The experimental lower bounds are $\kappa_{\mathrm{n}} r_{0}=.28(m=3)$ and $\kappa_{\mathrm{n}} r_{0}=.42(m=5)$, see table 1 . This is consistent with the values $K_{\text {rib }}^{3,2}=-.17$ $(m=3)$ and $K_{\mathrm{rib}}^{5,4}=.36(m=5)$ found in the linear stability analysis for $\nu=.3$, see table 3 Note that the bifurcation diagram predicts that a subcritical pitchfork bifurcation there, so these values are indeed the stability limits.

\section{Conclusion}

We presented experiments using metallic strips that had acquired residual curvature after being deformed plastically. These strips were formed into a loop shape, and then folded into multiply covered loops with a covering index equal to $m=1$ (single loop), $m=3$ and $m=5$. Buckling instabilities were observed, both when the ratio of the natural curvature to the curvature of the loop is small (overcurved case) and when it is large (undercurved case).

Motivated by these experiments, we carried out stability analyses for elastic ribbons. We addressed the linear stability problem near $m$-covered circular configurations, and computed non-linear equilibrium branches numerically. We used the mathematical formulation of Dias and Audoly [3], who treat an elastic ribbon as a kinematically constrained rod governed by non-linear constitutive laws. This formulation allowed us to analyze the ribbon and rod models in a unified language, and to easily adapt stability analyses routinely performed for elastic rods to ribbons. By a detailed comparison of the models, we highlighted a number of differences in their stability properties.

Many of these differences were interpreted, based on a simple feature of naturally curved ribbons, which has not been reported so far: the tangent twisting modulus can become negative when a ribbon is undercurved. This change of sign induces buckling instabilities whose existence has been confirmed by finite element simulations of an elastic shell having a small height-to-radius ratio.

As a final remark, we would like to emphasize that these buckling instabilities are different from the classical lateral torsional buckling instabilities of anisotropic beams: the latter can be observed for linearly elastic rods, and they make use of twisting to transfer a bending moment initially applied in the stiffer direction (i.e. involving the larger principal bending modulus) onto the weaker direction (i.e. involving the smaller principal bending modulus); by contrast, the buckling instabilities for ribbons which we described in this paper require the twisting modulus to be non-constant, and have been demonstrated in geometries where the initial bending moment is along the weaker direction.

\section{References}

[1] Antman, S.: Nonlinear problems of elasticity, $2^{\text {nd }}$ edn. Springer (2005) 
[2] Dias, M.A., Audoly, B.: A non-linear rod model for folded elastic strips. Journal of the Mechanics and Physics of Solids 62, 57-80 (2014)

[3] Dias, M.A., Audoly, B.: "Wunderlich, meet Kirchhoff": A general and unified description of elastic ribbons and thin rods. Journal of Elasticity (2014)

[4] Doedel, E.J., Champneys, A.R., Fairgrieve, T.F., Kuznetsov, Y.A., Sandstede, B., Wang, X.J.: AUTO-07p: continuation and bifurcation software for ordinary differential equations. See http://indy.cs.concordia.ca/auto/ (2007)

[5] Goriely, A.: Twisted elastic rings and the rediscoveries of Michell's instability. Journal of Elasticity 84, 281-299 (2006)

[6] Goriely, A., Shipman, P.: Dynamics of helical strips. Physical Review E 61(4), 4508-4517 (2000)

[7] Haijun, Z., Zhong-can, O.Y.: Spontaneous curvature-induced dynamical instability of Kirchhoff filaments: Application to DNA kink deformations. Journal of Chemical Physics 110, 1247 (1999)

[8] Hibbitt, D., Karlsson, B., Sorensen, P.: Abaqus manual version 6.11. Tech. rep., Dassault Systèmes (2011)

[9] Hinz, D.F., Fried, E.: Translation and interpretation of Michael Sadowsky's paper "Theory of elastically bendable inextensible bands with applications to the Möbius band". Journal of Elasticity pp. 1-11 (2014)

[10] Hinz, D.F., Fried, E.: Translation of Michael Sadowsky's Paper “An Elementary Proof for the Existence of a Developable Möbius Band and the Attribution of the Geometric Problem to a Variational Problem". Journal of Elasticity (2014)

[11] Hinz, D.F., Fried, E.: Translation of Michael Sadowsky's paper "The differential equations of the Möbius Band". Journal of Elasticity pp. 1-4 (2014)

[12] Hoffman, K.A.: Methods for determining stability in continuum elastic-rod models of DNA. Phil. Trans. R. Soc. Lond. A 362 (2004)

[13] Hoffman, K.A., Manning, R.S., Maddocks, J.H.: Link, twist, energy and the stability of DNA minicircles. Biopolymers 70(2), 145-157 (2003)

[14] Keller, H.B.: Numerical solution of bifurcation and nonlinear eigenvalue problems. In: P.H. Rabinowitz (ed.) Applications of Bifurcation Theory, pp. 359-384. Academic Press (1977)

[15] Manning, R.S., Hoffman, K.A.: Stability of $n$-covered circles for elastic rods with constant planar intrinsic curvature. Journal of elasticity 62(1), $1-23(2001)$ 
[16] Michell, J.H.: On the stability of a bent and twisted wire. Messenger of Mathematics 11, 181-184 (1889-1890)

[17] Press, W.H., Teukolsky, S.A., Vetterling, W.T., Flannery, B.P.: Numerical recipes, $3^{\text {rd }}$ edn. Cambridge University Press (2007)

[18] Sadowsky, M.: Die Differentialgleichungen des Möbiusschen Bandes. In: Jahresbericht der Deutschen Mathematiker-Vereinigung, vol. 39 (2. Abt. Heft 5/8), pp. 49-51 (1929)

[19] Sadowsky, M.: Ein elementarer Beweis für die Existenz eines abwickelbaren Möbiusschen Bandes und die Zurückführung des geometrischen Problems auf ein Variationsproblem. In: Sitzungsberichte der Preussischen Akademie der Wissenschaften, physikalisch-mathematische Klasse, 17. Juli 1930, Mitteilung vom 26. Juni, pp. 412-415 (1930)

[20] Sadowsky, M.: Theorie der elastisch biegsamen undehnbaren Bänder mit Anwendungen auf das Möbiussche Band. In: Proceedings of the 3rd International congress of applied mechanics, vol. 2, pp. 444-451. Stockholm (1930)

[21] Seffen, K.A., Pellegrino, S.: Deployment dynamics of tape springs. Proceedings of the Royal Society of London. Series A: Mathematical, Physical and Engineering Sciences 455(1983), 1003-1048 (1999)

[22] Starostin, E.L., van der Heijden, G.H.M.: The shape of a Möbius strip. Nature Materials 6(8), 563-567 (2007)

[23] Starostin, E.L., van der Heijden, G.H.M.: Tension-induced multistability in inextensible helical ribbons. Physical Review Letters 101, 084,301 (2008)

[24] Thompson, J.M.T., Champneys, A.R.: From helix to localized writhing in the torsional post-buckling of elastic rods. Proceedings of the Royal Society A: Mathematical, Physical and Engineering Sciences 452(1944), 117-138 (1996)

[25] Todres, R.E.: Translation of W. Wunderlich's "on a developable Möbius band". Journal of Elasticity pp. 1-12 (2014)

[26] Wunderlich, W.: Über ein abwickelbares Möbiusband. Monatshefte für Mathematik 66(3), 276-289 (1962) 